\title{
PRELIMINARY DATA REPORT FOR THE SAN JUAN BASIN-CROWNPOINT SURVEILLANCE STUDY.
}

BY PETER F. FRENZEL, STEVEN! D. CRAIGG, AND ELIZABETH T. PADGETT

OPEN-FILE REPORT $81-484$

Prepared in cooperation with the U.S. Bureau of Indian Affairs

Albuquerque, New Mexico

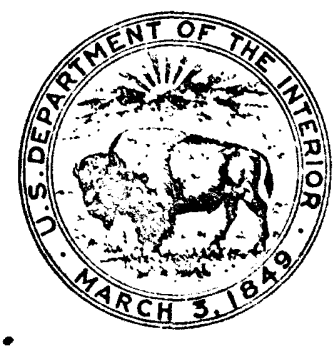


$\therefore$
UNITED STATES DEPARTMENT OF THE INTERIOR

JAMES G. WATT, Secretary

GEOLOGICAL SURVEY

Doyle G. Frederick, Acting Director

For additional information write to:

District Chief

U.S. Geological Survey Water Resources Division P.0. Box 26659

Albuquerque, New Mexico 87125
For sale by:

Open-File Services Section Branch of Distribution

U.S. Geological Survey, MS 306

Box 25425, Denver Federal Center Denver, Colorado 80225

(303) 234-5888 
Page

Figure 1. Map showing structural elements of the San Juan Basin and

location of the study area

2. We11-numbering systems

3. Graph showing summary of water-level altitudes recorded in the Crownpoint Morrison observation well (17.12.17.3333) -- 7

4. Map showing reported values of transmissivity and storage coefficient for the Westwater Canyon Member of the Morrison Formation in the vicinity of Crownpoint, New Mexico - - -

\section{TABLES}

Table 1. Records of wells in San Juan Basin observation network

2. Major chemical constituents of water :rom selected wells in the vicinity of Crownpoint, Jew Mexico

3. Minor chemical constituents of water from selected

wells in the vicinity of Crownpoint, New Mexico

\section{IN IH-POUND TO METRIC UNIT CONVERSION FACTORS.}

In this repc't measurements of distances and transmissivities are given in inch-pourl units only. The following table contains factors for converting to metric units.

Multiply inch-pound units

foot ( $f t$ )

foot squared per day $\left(f t^{2} / d\right)$ mile (mi)
By

0.3048

0.0929

1.609
To obtain metric units

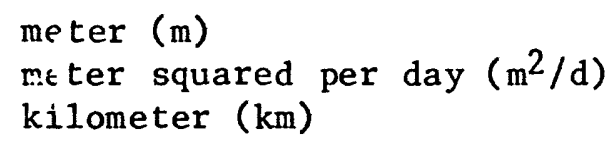

National Geodetic Vertical Datum of 1929 (NGVD of 1929): A geodetic datum derived from a general adjustment of the first-order level nets of both the United States and Canada, formerly called "Mean Sea Level." NGVD of 1929 is referred to as sea level in this report. 


\title{
PRELIMINARY DATA REPORT FOR THE
}

SAN JUAN BASIN-CROWNPOINT SURVEILLANCE STUDY

By Peter F. Frenzel, Steven D. Craigg, and Elizabeth T. Padgett

\begin{abstract}
Geohydrologic data that may be used to predict the effects of mining on Navajo water resources in the San Juan structural basin are reported as wel1 as the current availability of data from other government agencies. Emphasis is on the vicinity of Crownpoint, New Mexico. A denser network of observation wells and more data on water quality, transmissivity, storage-coefficient, and aquifer discharge would be useful for further studies in this area.
\end{abstract}

\section{INTRODUCTION}

Purpose and scope

Most of the conmunities in the southern and western parts of the San Juan structural basin (fig. 1) rely on ground water. Ground-water supplies may be adversely af iected by uranium mining, esfecially at Crownpoint where the main aquifer (the Westwater Canyon Member of the Morrison Formation) contains uranium ore and might be partly dewatered by mines in the vicinity.

The Water Resources Division (WRD) of the U.S. Geological Survey has, undertaken a 2-year project in cooperation with the Eastern Navajo Agency of the Bureau of Indian Affairs (BIA) to provide a hydrologic data base and observation-we11 net vork for the San Juan structural basin. An area within a 20-mile radius of $\mathrm{C}$.:ownpoint is of immediate concern. The emphasis of this study is on the rejatively extensive sandstone aquifers that contain water under artesian cond $i$ :ions.

This report is intended to present pertinent geohydrologic data that are currently available, to indicate which agencies are collecting such data, and to point out additicnal data needs. These data, although not complete, will be useful in estima:ing the hydrologic impact of impending mine development on the water supply of Crownpoint.

The geology and hydrology of the study area are beyond the scope of this report; they are discussed in general terms by Lyford (1979). 


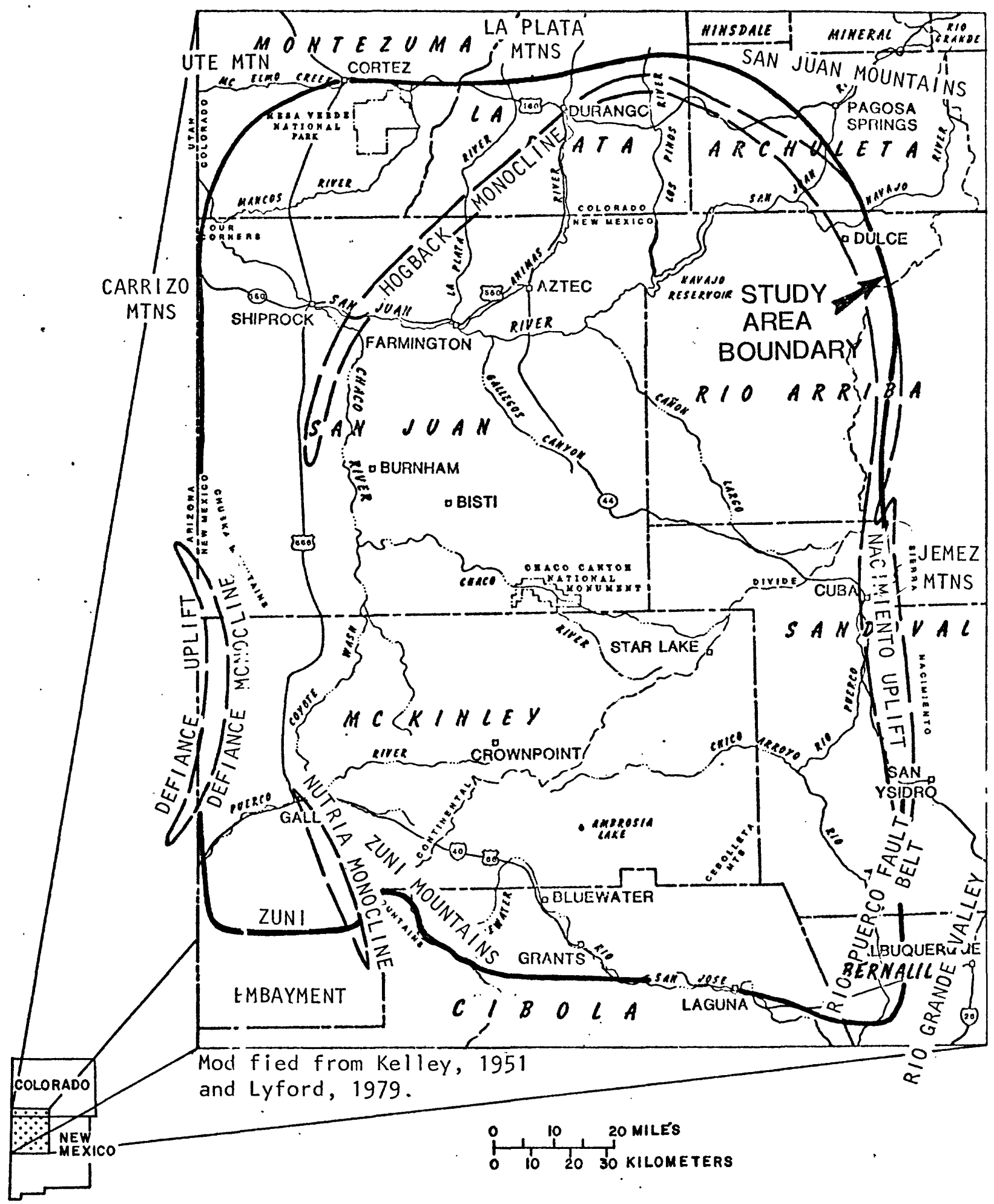

Figure 1. Structural elements of the San Juan Basin and location of the study area 
Two numbering systems are used in this report to locate a well. The first uses the common subdivision of lands into townships, ranges, and sections. In this system, the location number is divided into four segments separated by periods. The first segment indicates the township north of the New Mexico Base Line, and the second denotes the range west of the New Mexico Principal Meridian. The third segment indicates the section within the township; and the fourth segment indicates the tract within which the well is located. To determine the fourth segment of the location number, the section is divided into quarters numbered $1,2,3$ and 4 for the $N W^{\frac{1}{4}}, N^{\frac{1}{4}}$, $S W^{\frac{1}{4}}$ and $\mathrm{SE}_{\frac{1}{4}}^{\frac{1}{4}}$ respectively. Each quarter section is further subdivided into as many as four subdivisions depending on how closely the well was located. As an example, a well with a location number 21.07 .28 .2134 is located in the southeast $\frac{1}{4}$ of the southwest $\frac{1}{4}$ of the northwest $\frac{1}{4}$ of the northeast $\frac{1}{4}$ of section 28, Township 21 North, Range 7 West.

A different numbering system is used for the main part of the Navajo Reservation. This area is divided into 15-minute quadrangles, each of which is assigned a number. The well number consis.s of the quadrangle number followed by the distance in miles from the east line and the distance in miles from the north line, in that order. Thus, a well numbered $32-3.65 \mathrm{x}$ 17.05 is in quadrangle number $32,3.65$ miles from the east line and 17.05 miles from the north line as shown in figurt: 2 .

\section{Acknowledgments}

The authors wculd like to thank the employees of other. government and tribal agencies $x d$ mining companies for their kindly and informative discussions. A spcsial word of appreciation is due the well owners who have allowed their well! to be used in the observation network and, in certain cases, have provided aquifer-test data. 


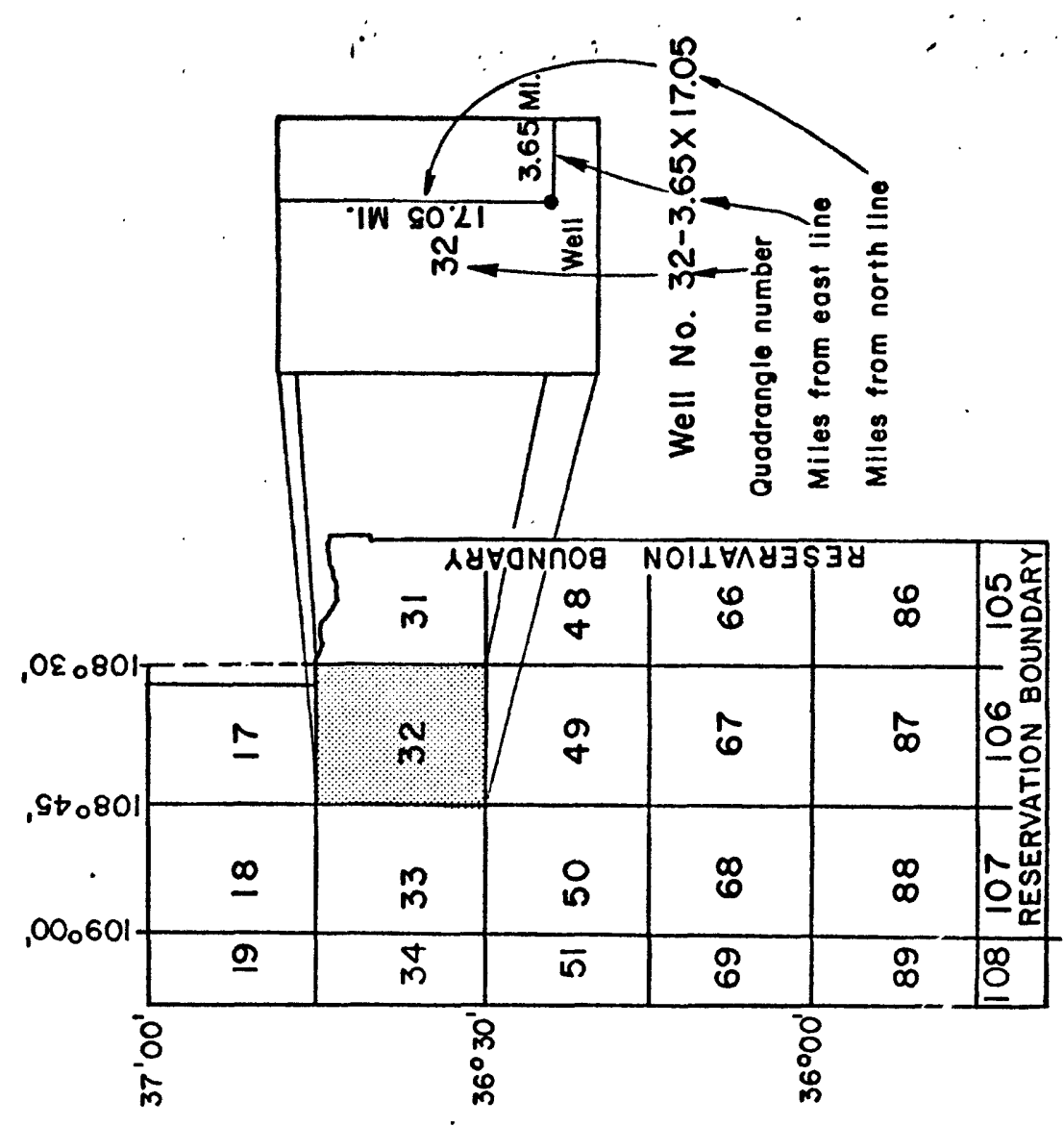

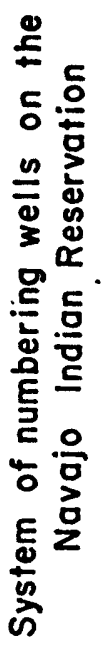

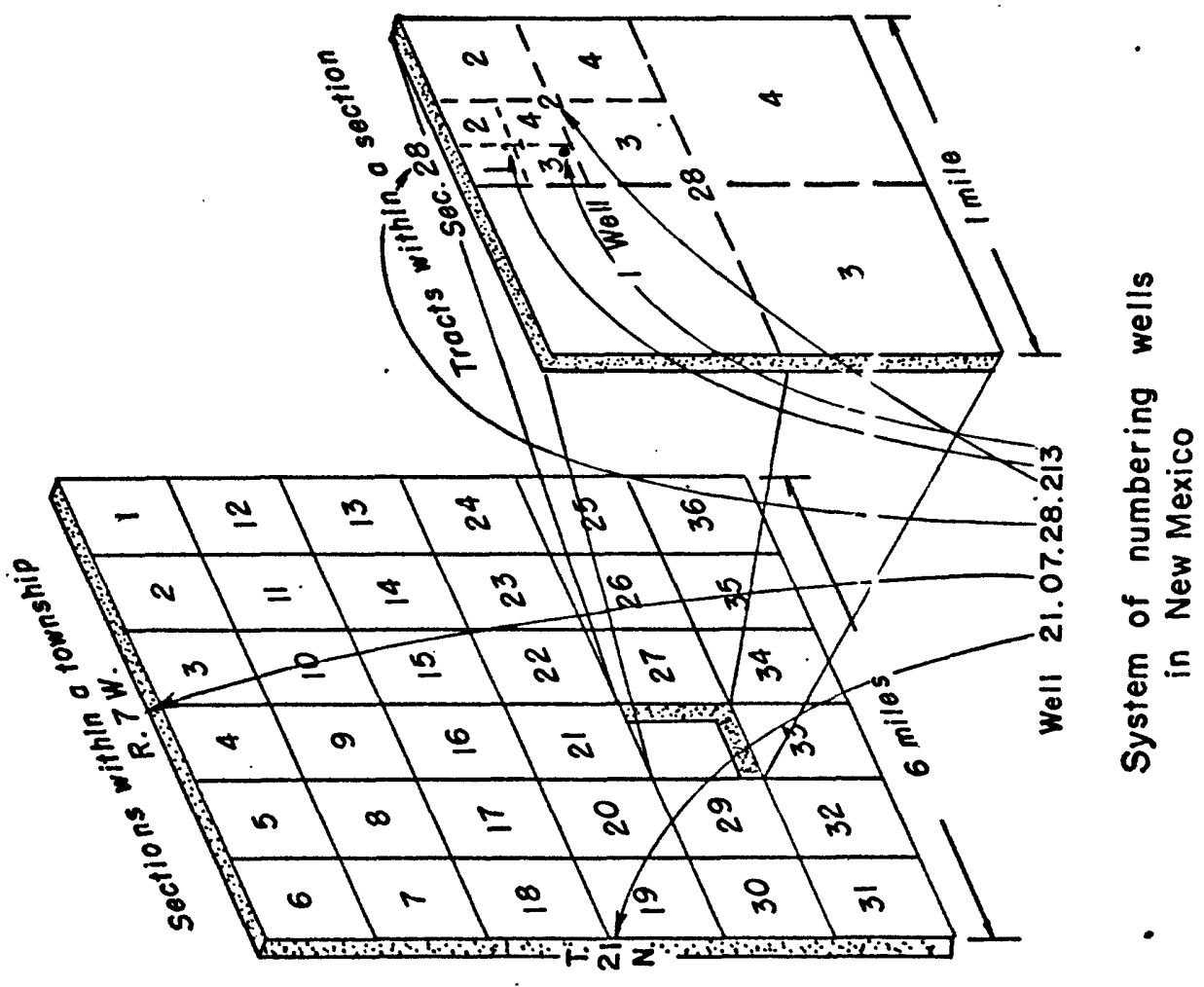




\title{
CURRENT AVAILABILITY OF DATA
}

\author{
Data collecting agencies
}

Currently at least 11 governmental and tribal agencies are collecting data that are pertinent to this study.

The New Mexico State Engineer office (SEO) collects data relating to well construction, aquifer tests, and, in certain cases, the volume of discharges at mines and wells. However, SEO data for most mines and wells currently operating (1981) are sparse.

The New Mexico Environmental Improvement Division (EID) has published reported historical mine-water discharges, as we11 as water-quality data (New Mexico Health and Environment Department, 1980). EID has an ongoing program of monitoring ground-water seepage from uranium operations. It also keeps records of reported data on in situ uranium-leaching projects near Crownpoint and is studying shallow ground water and surface water in uranium producing areas. Data collected by these programs probably will be pertinent to long term water-quality considerations for the deep aquifers.

The New Mexico Energy and Minerals Department has published 1978 mine-water discharge rates (Perkins, 1979) and is present1y revising that publication. The Mining and Minerals Division of the Energy and Minerals Department keeps records of water-quality and aquifer-test data for aquifers associated with strip mining. The aquifers most often involved are sandstones of the Mesaverde Group.

The U.S. Envirınmental Protection Agency (EPA) maintains files of data reported under National Pollutant Discharge Elimination System (NPDES) regulations. These data are on file in Dallas, 'exas, and copies are on file with EID in Santa Fe. These data may be usefui as a cross-check on discharge data for mines. However, the amount of liquid discharged. from mining and milling operations is generally not directly related to the amount of ground-water inflow to the mines.

The BIA, U.S. Public Health Service, Navajo Water and Sanitation Department, Navajo Tribal Utility Authority (NTUA), and City of Gallup own and maintain wells and water systems in the area. They provided WRD with well records, well-discharge rates, water-level measurements, and aquifertest records. The BIA Soil, Water, and Materials Testing Laboratory at Gallup provided many of the water-quality analyses presented in this report.

The Conservation Division of the U.S. Geological Survey is responsible for certain Federal and Indian interests. The Conservation Division, as part of its uranium-mine permitting operations, requires the collection of water-quality and aquifer test data, as well as programs to monitor water quality and hydraulic head. 


\section{Presentation of data}

Water-level altitudes measured in wells that are part of the observation-we11 network are shown in figure 3 and plates 1-5. This network consists of selected wells in which water levels are measured periodically, in most cases annually. Measurements will be made for the foreseeable future. Reported values of transmissivity and coefficient of storage for the Westwater Canyon Member of the Morrison Formation are shown in figure 4.

Records of wells in the observation-well network are shown in table 1. The major chemical constituents of water from selected wells in the vicinity of Crownpoint, New Mexico, are shown in table 2. Data for each well appear on facing pages. Minor chemical constituents are shown in table 3 .

\section{NEEDS FOR ADDITIONAL DATA}

The large open circles in plates 1-3 indicate the general areas where more wells would be useful in those aquifers that are presently of particular concern. A similar density of observation wells may be useful in the future for other aquifers (plates 4 and 5). Efforts to build these networks using existing wells will continue.

More baseline rater-quality data are needed, especially with regard to minor constituents. The regional observation well network may be used to obtain water șamplrs for chemical analyses. Sampling, however, will be selective because of the high cost of chemical anilyses.

Additional transmissivity and storage-coeficient data are needed for all of the aquifers in the study area. The pr'mary source of these costly data will be from other agencies and companies rather than actual testing by the Water Resources Division.

Certain aquifer discharge data are not presently being systematically recorded by any government agency. This is generally the case with schools, public supply systems, and certain large industrial establishments. An effort will be made to solicit these data. 


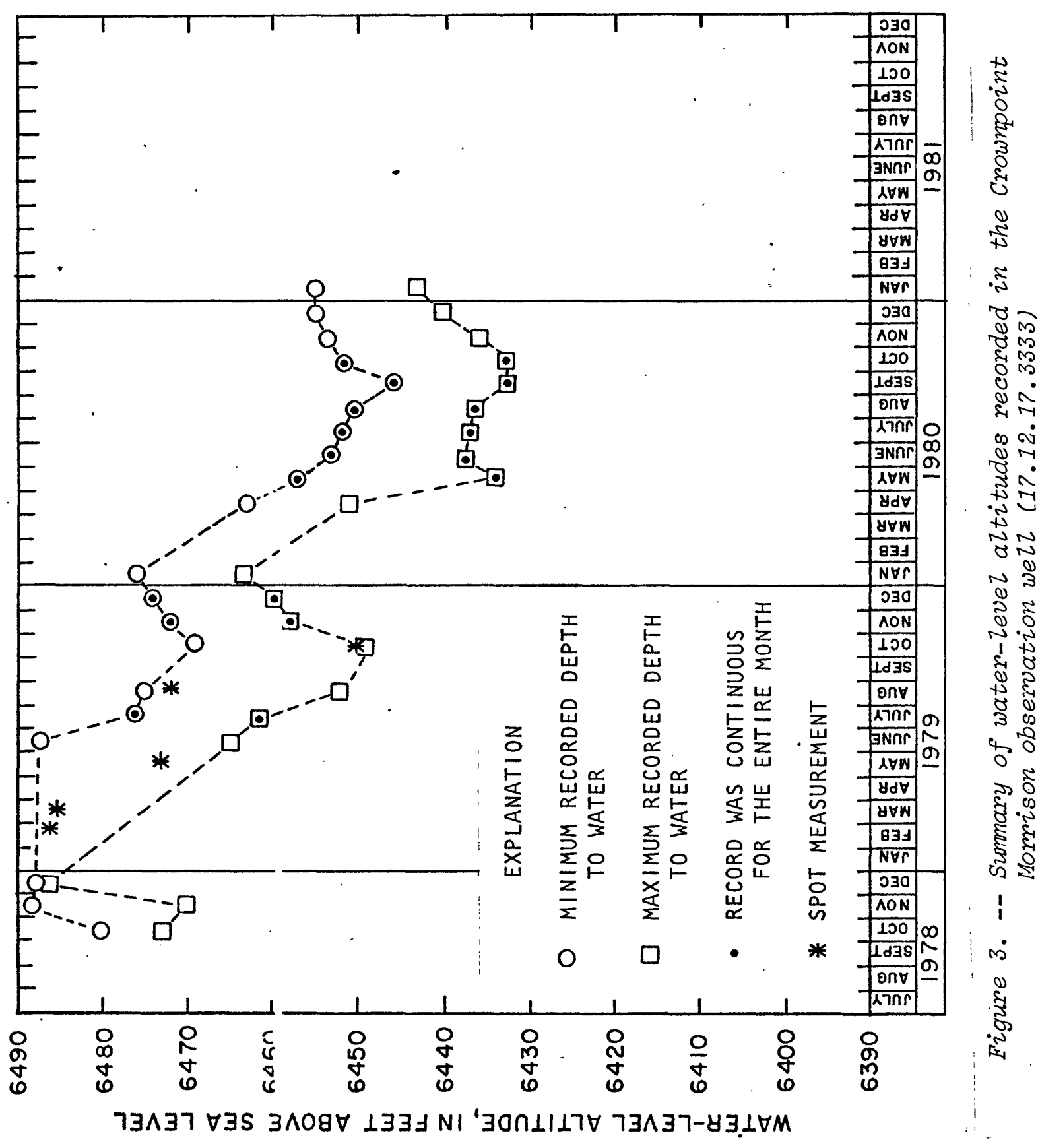




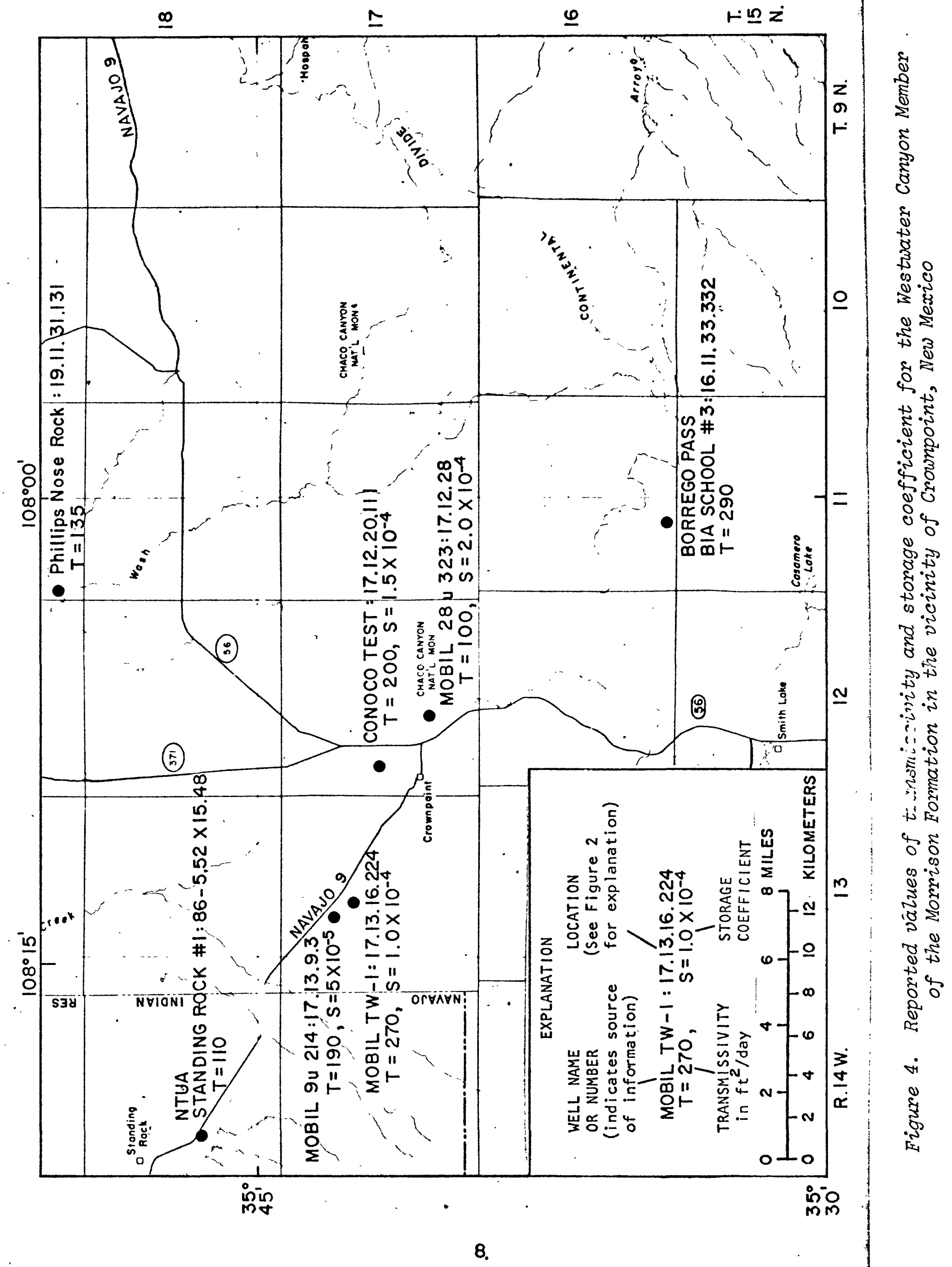




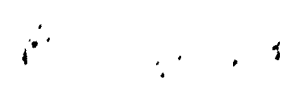

\section{REFERENCES}

Kelly, V. C., 1951, Tectonics of the San Juan Basin, in Guidebook of the south and west sides of the San Juan Basin, New Mexico and Arizona: New Mexico Geological Society, 2nd Field Conference, p. $124-130$.

Lyford, F. P., 1979, Ground water in the San Juan Basin, New Mexico and Colorado: U.S. Geological Survey Water Resources Investigations 79-73, 22 p., 11 figs.

New Mexico Health and Environment Department, 1980, Water-quality data for discharges from uranium mines and mills in New Mexico: Environmental Improvement Division, Water Pollution Control Bureau, Santa Fe, 87 p., 13 figs.

Perkins, B. L., 1979, An overview of the New Mexico uranium industry: New Mexico Energy and Minerals Department, Santa Fe, 144 p. 


\section{$\therefore \quad \therefore \quad$ i}

Table 1. Records of wells in San Juan Basin obsemation network

Location.--The location of a well or spring is described by using the system of quartering by sections or the Navajo system for the Navajo Reservation. The systems are explained in the text (fig. 2). All locations are defined as accurately as possible with the information available.

Number or name.--The number or name assigned to a well may be the owner's name or number, the BIA or Navajo name or number, a traditional name, or the name of a nearby landmark.

Depth.--Depth is the total depth of a well in feet below land surface as measured (M) by U.S. Geological Survey or reported by other sources.

Altitude.--Altitude of the land surface above sea level (in feet) at the we11.

Depth to water.--Depth to static water level below the land surface (in feet). Values with decimal point accuracy wfre measured, others reported $(R)$, or questionable $(Q)$. A plus sign $(t)$ signifies the water level is above the land surface. " $F$ " indica:es the well was flowing on the date given.

Water-level altitude--Altitude of water surface above sea level (in feet), rounded to the nearest foot. Reported values are indicated by $(R)$, and questionable values by $(Q)$. A plus sign $(t)$ indicates the water level was greater than the value given.

Date.--The date given is that of the water-1evel measurement noted on the same line.

Producing interval.--Producing interval is the portion(s) of the well that is open to the water-bearing unit, in feet below land surface.

Principle water-bearing unit(s).--The geologic unit or units from which the well obtains water, recorded in order of importance. Questionable units are followed by (?). The abbreviations used for water-bearing units are as follows:

Tertiary:

$$
\begin{aligned}
& \text { Tsj - San Jose Formation } \\
& \text { Tsj1 - Llaves Member } \\
& \text { Tn - Nacimiento Formation }
\end{aligned}
$$

Tertiary-Cretaceous:

TKoa - Ojo Alamo Sandstone 


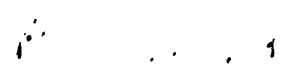

Table 1. Records of wells in San Juan Basin observation network - Continued

Cretaceous:

$$
\begin{aligned}
& \text { Kpc - Pictured Cliffs Sandstone } \\
& \text { Kch - Cliff House Sandstone } \\
& \text { Kmf - Menefee Formation } \\
& \text { Kpl - Point Lookout Sandstone } \\
& \text { Kg - Gallup Sandstone } \\
& \text { Kd - Dakota Sandstone }
\end{aligned}
$$

Jurassic:

$$
\begin{aligned}
& \text { Jm - Morrison Formation } \\
& \text { Jmw - Westwater Canyon Member } \\
& \text { Jmr - Recapture Member } \\
& \text { Jcs - Cow Springs Sandstone } \\
& \text { Je - Entrada Sandstone }
\end{aligned}
$$

Triassic:

TRc - Chinle Formation

TRes - Shinarump Conglomerate

Permian:

Psa - San Andres Limestone

Pg - Glorieta Sandstone 
Tabie 1. Records of wells in San Juan Basin observation network - Continued

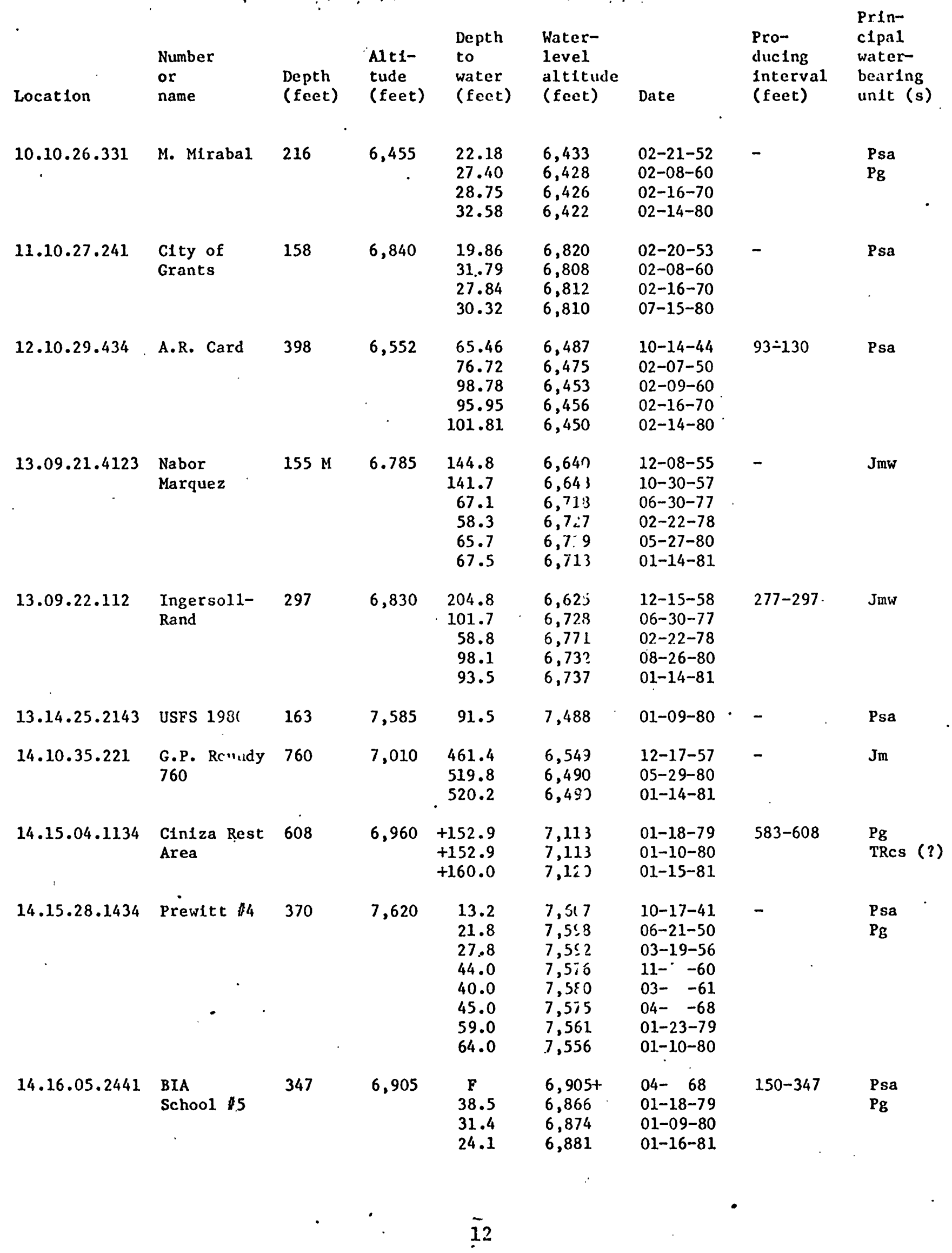


Table 1. Records $\rho f$ wells in San Juan Basin observation network - Continued

\begin{tabular}{|c|c|c|c|c|c|c|c|c|}
\hline Location & $\begin{array}{l}\text { Number } \\
\text { or } \\
\text { name }\end{array}$ & $\begin{array}{l}\text { Depth } \\
\text { (feet) }\end{array}$ & $\begin{array}{l}\text { Alt1- } \\
\text { tude } \\
\text { (feet) }\end{array}$ & $\begin{array}{l}\text { Depth } \\
\text { to } \\
\text { water } \\
\text { (feet) }\end{array}$ & $\begin{array}{l}\text { Water- } \\
\text { level } \\
\text { altitude } \\
\text { (feet) }\end{array}$ & Date & $\begin{array}{l}\text { Pro- } \\
\text { ducing } \\
\text { Interval } \\
\text { (feet) }\end{array}$ & $\begin{array}{l}\text { Prin- } \\
\text { cipal } \\
\text { water- } \\
\text { bear1ng } \\
\text { unit (s) }\end{array}$ \\
\hline 15.14 .09 .233 & $16 \mathrm{~T}-587$ & 1,334 & 7,520 & $\begin{array}{l}448 \mathrm{R} \\
442.2 \\
380.1\end{array}$ & $\begin{array}{l}7,072 \mathrm{R} \\
7,078 \\
7,140\end{array}$ & $\begin{array}{l}02-09-76 \\
06-11-80 \\
01-14-81\end{array}$ & $\begin{array}{l}162-243 \\
466-507 \\
1,201-1334\end{array}$ & $\mathrm{Je}$ \\
\hline 15.17 .24 .4121 & $\begin{array}{l}\text { Ft. Wingate } \\
69\end{array}$ & 1,350 & 6,680 & $\begin{array}{r}+29.0 \\
+35.5 \\
+40.2\end{array}$ & $\begin{array}{l}6,709 \\
6,715 \\
6,720\end{array}$ & $\begin{array}{l}01-18-79 \\
01-09-80 \\
01-10-81\end{array}$ & $1,000-1,125$ & Psa \\
\hline 15.18 .16 .1113 & Ga11up 14 & - & 6,515 & $\begin{array}{l}304.5 \\
299.0 \\
286.4\end{array}$ & $\begin{array}{l}6,210 \\
6,216 \\
6,229\end{array}$ & $\begin{array}{l}01-19-79 \\
01-10-80 \\
01-15-81\end{array}$ & - & $\mathbf{K}_{\mathbf{g}}$ \\
\hline 16.04 .06 .331 & • & 250 & 6,235 & $\begin{array}{l}93.2 \\
93.6 \\
92.9 \\
93.2\end{array}$ & $\begin{array}{l}6,142 \\
6,141 \\
6,142 \\
6,142\end{array}$ & $\begin{array}{l}08-31-78 \\
07-17-79 \\
06-18-80 \\
01-18-81\end{array}$ & $128-245$ & Kpl \\
\hline 16.04 .18 .4444 & $\begin{array}{l}\text { NMBBMMR } \\
R-21\end{array}$ & 244 & 6,395 & $\begin{array}{r}102.2 \\
98.8 \\
102.5 \\
101.9 \\
101.2\end{array}$ & $\begin{array}{l}6,293 \\
6,296 \\
6,293 \\
6,293 \\
6,294\end{array}$ & $\begin{array}{l}07-27-78 \\
08-29-78 \\
07-17-79 \\
06-18-80 \\
01-18-81\end{array}$ & $84-241$ & Kpl \\
\hline 16.04 .36 .2321 & $\begin{array}{l}\text { BLAt } \\
\text { Homestake }\end{array}$ & 602 & 6,165 & $\begin{array}{l}+214.3 \\
+178.5 \\
+135.8 \\
+155.4 \\
+160.0 \\
+168.1\end{array}$ & $\begin{array}{l}6,379 \\
6,343 \\
6,301 \\
6,320 \\
6,325 \\
6,33\end{array}$ & $\begin{array}{l}10-04-74 \\
04-13-78 \\
07-22-80 \\
08-06-80 \\
10-19-80 \\
01-18-81\end{array}$ & $\begin{array}{l}410-420 \\
468-469\end{array}$ & $\mathbf{K g}$ \\
\hline 16.05 .02 .444 & NMBBMR R2 & 250 & 6,310 & $\begin{array}{l}123.0 \\
120.7 \\
118.9 \\
117.3 \\
120.3\end{array}$ & $\begin{array}{l}6,1 \times 7 \\
6,18) \\
6,191 \\
6,193 \\
6,190\end{array}$ & $\begin{array}{l}10-13-78 \\
05-23-79 \\
07-17-79 \\
06-18-80 \\
01-18-81\end{array}$ & $200-240$ & Kp1 \\
\hline 16.11 .17 .4322 & $15 \mathrm{~T}-505$ & 570 & 7,070 & $\begin{array}{l}265.1 \\
295.6 \\
280.9 \\
308.7\end{array}$ & $\begin{array}{l}6,805 \\
6,774 \\
6,789 \\
6,761\end{array}$ & $\begin{array}{l}07-10-59 \\
02-23-78 \\
05-28-80 \\
01-15-81\end{array}$ & $470-570$ & $\mathrm{~K}_{\mathbf{g}}$ \\
\hline 16.11 .33 .332 & Borr. Pass & 2,023 & 7,350 & $\begin{array}{l}752 \mathrm{R} \\
756 \mathrm{R} \\
600 \mathrm{R}, \mathrm{Q}\end{array}$ & $\begin{array}{l}6,598 R \\
6,594 R \\
6,750 R, Q\end{array}$ & $\begin{array}{l}09-08-72 \\
06-10-77 \\
05-28-80\end{array}$ & $1,801-2,023$ & Jmw \\
\hline 16.16 .15 .4322 & $16 \mathrm{~T}-513$ & $318 \mathrm{M}$ & 6,850 & $\begin{array}{l}182.0 \\
213.0 \\
160 Q \\
302.0 \\
308.0 \\
312.3\end{array}$ & $\begin{array}{l}6,693 \\
6,662 \\
6,7150 \\
6,573 \\
6,567 \\
6,563\end{array}$ & $\begin{array}{l}07-27-59 \\
10-05-76 \\
06-21-77 \\
02-24-78 \\
06-10-80 \\
01-15-81\end{array}$ & $206-318$ & $\begin{array}{l}\mathrm{Jmw} \\
\mathrm{Kd}(?)\end{array}$ \\
\hline
\end{tabular}


Table 1. Records ofiwezls in San sian Basin observation network - Continued

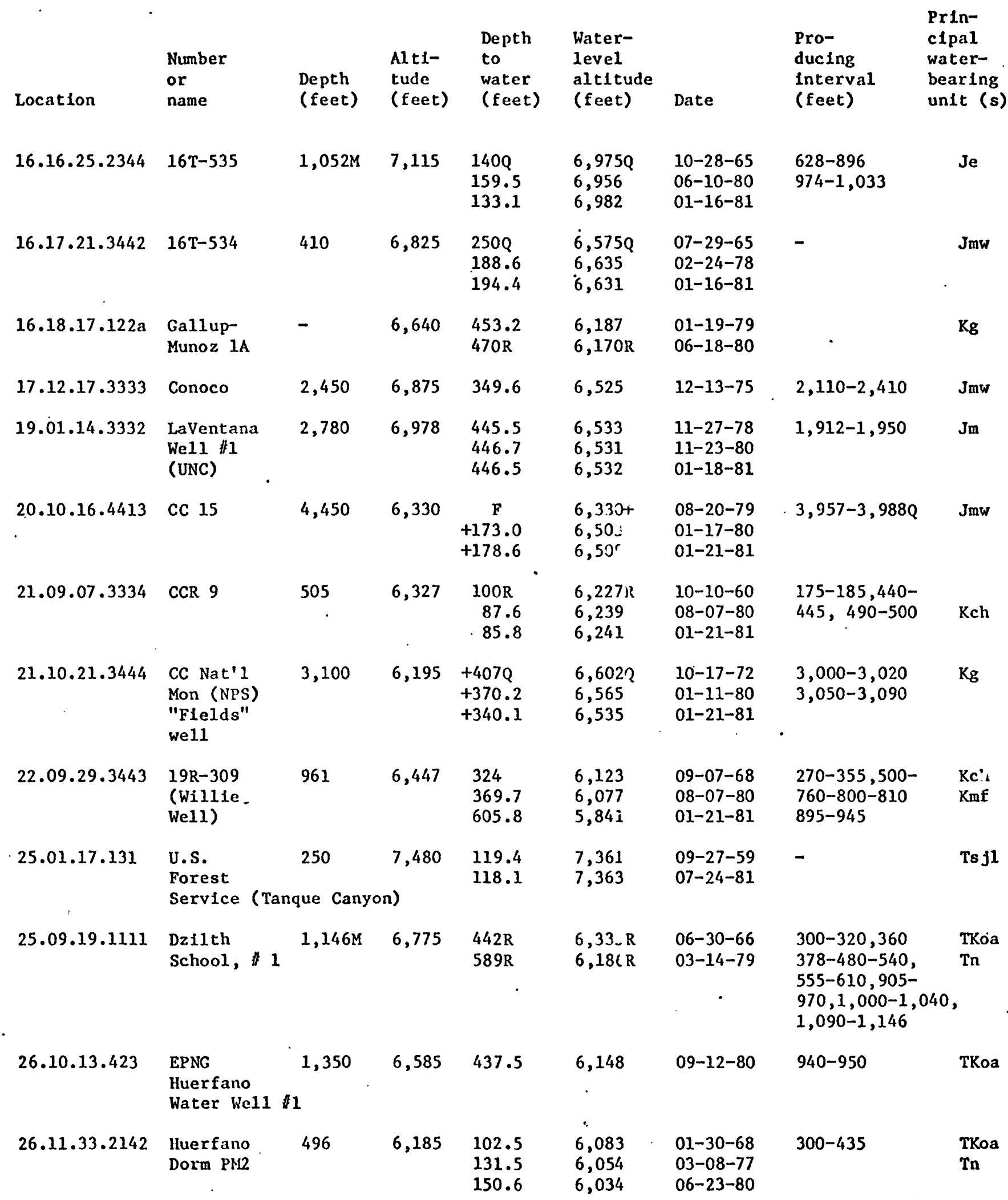


Tabie 1. Records of ve.lz in San Juan Basin observation network - Concluded

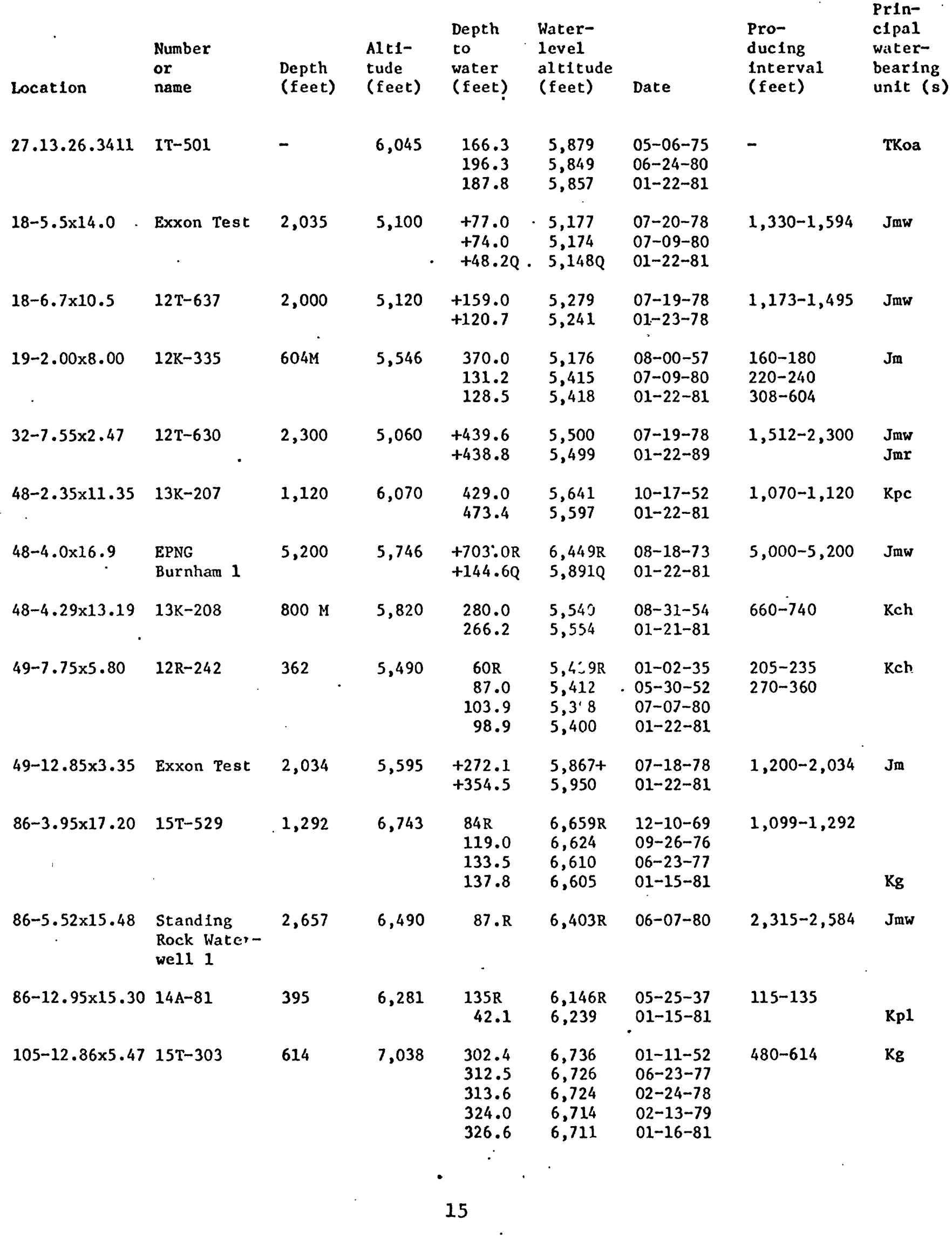


还

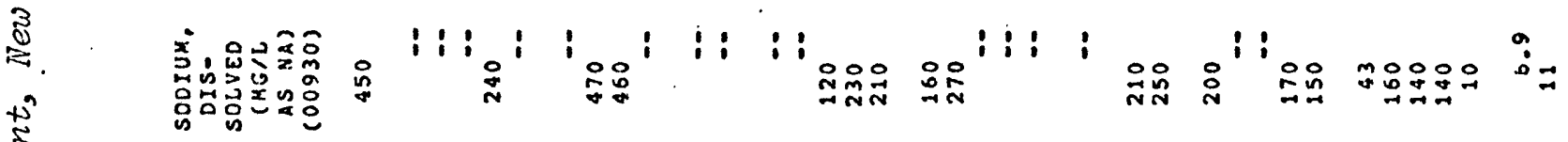

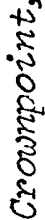

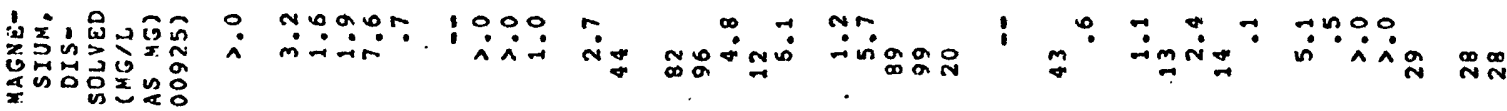

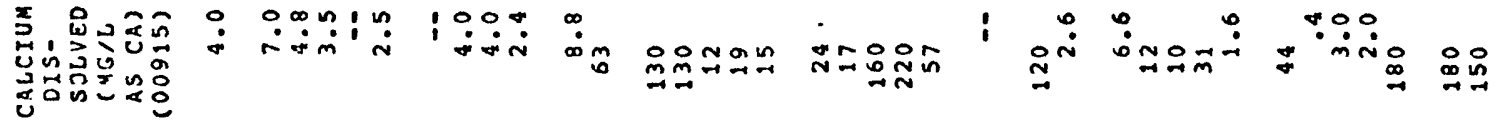
त)

$\frac{5}{.4}$

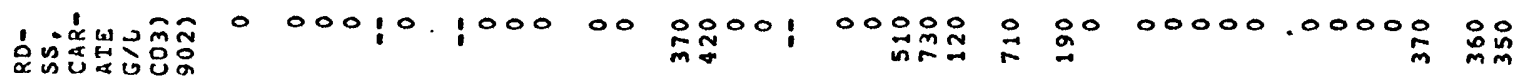

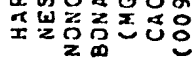

$\frac{\pi}{4}$

.5

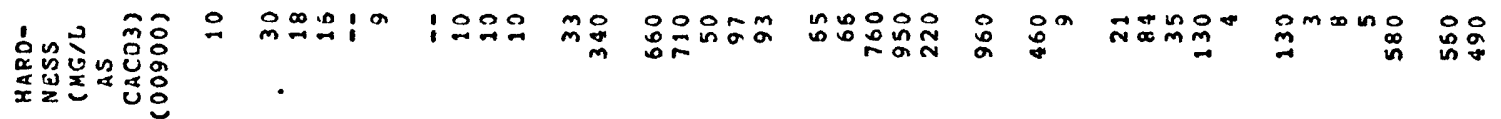

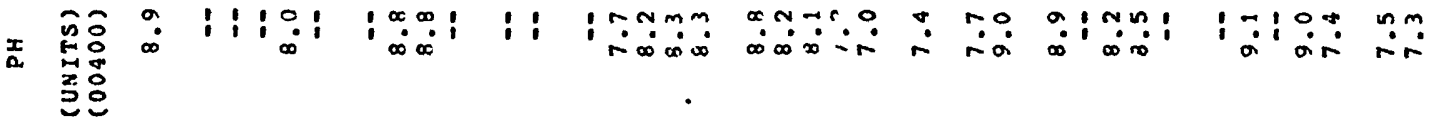

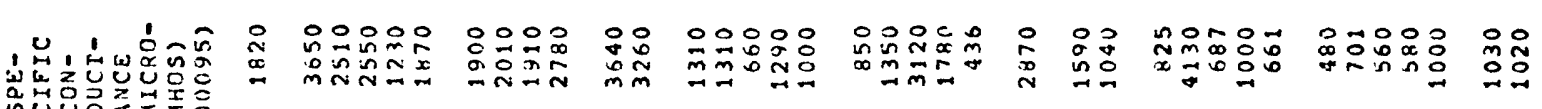

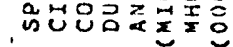

苾

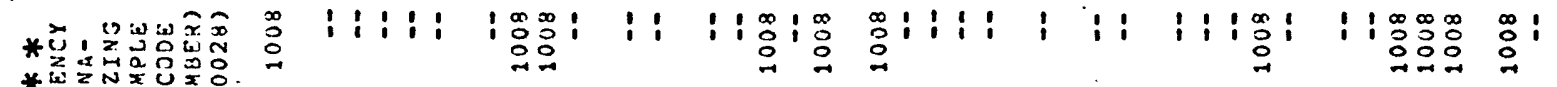
*

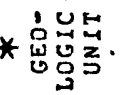

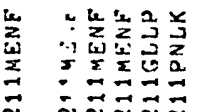

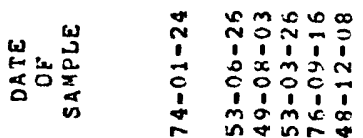

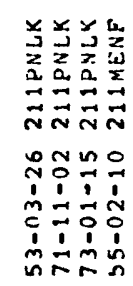

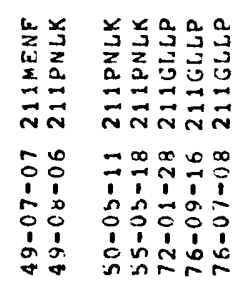

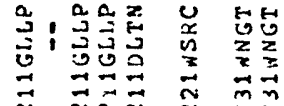

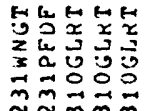

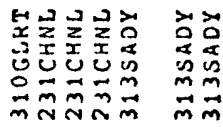

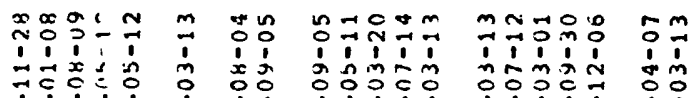

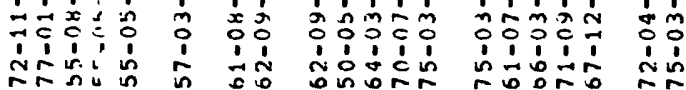
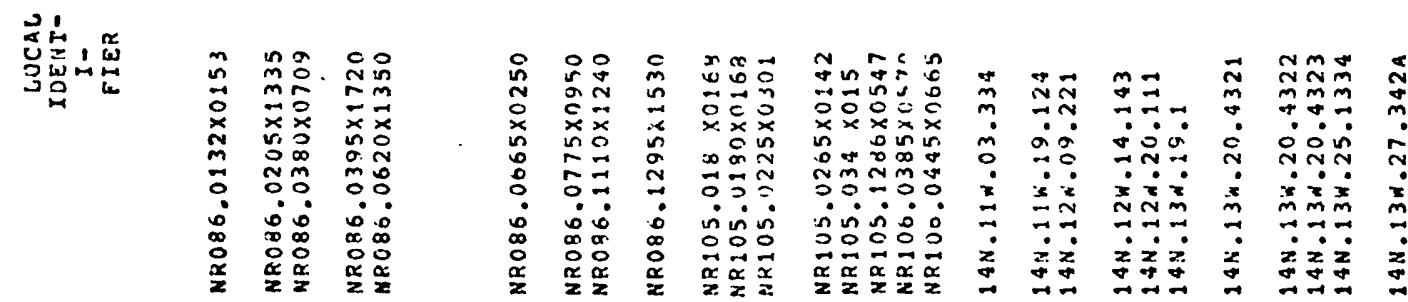

$\dot{0}$

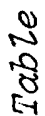




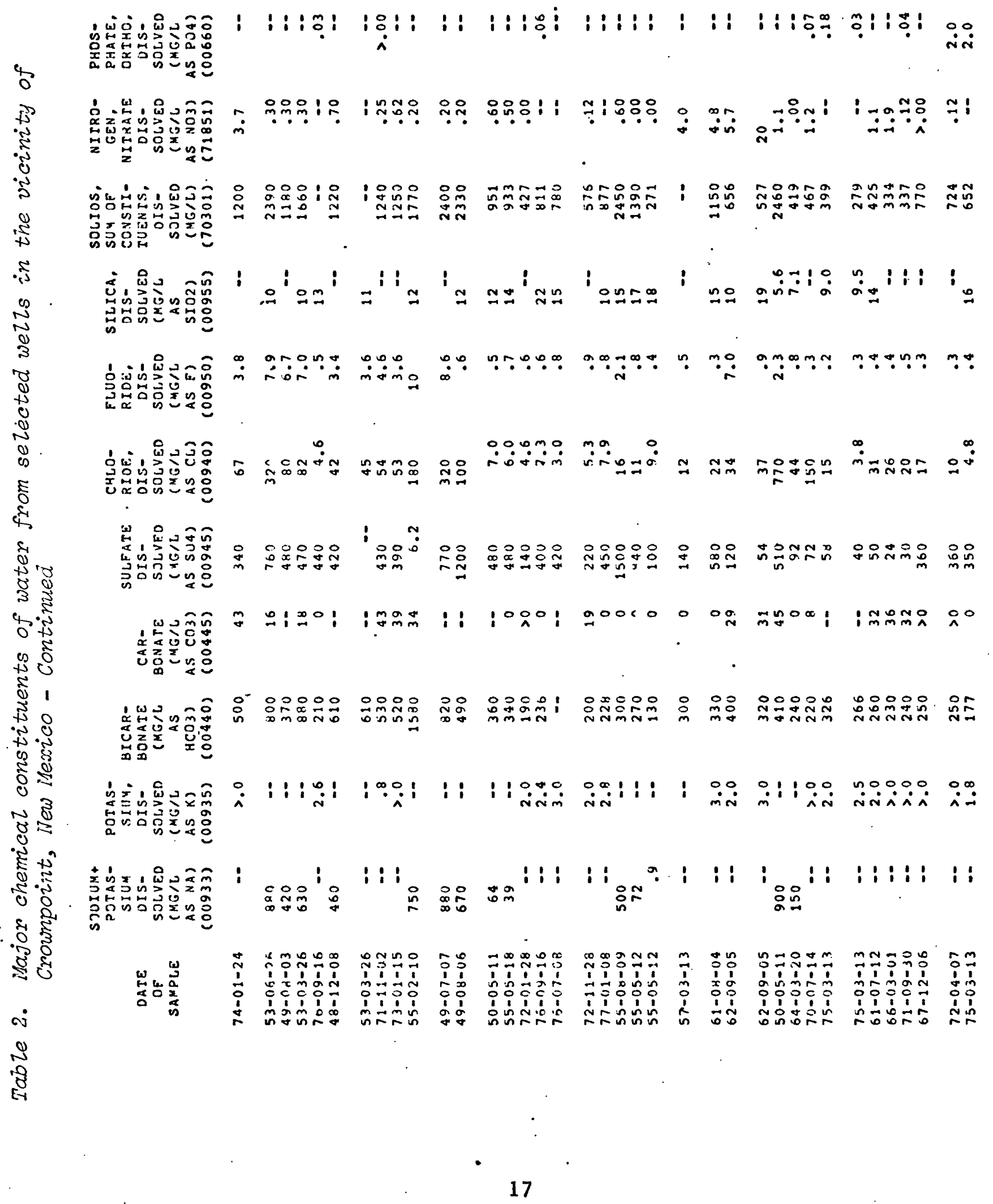




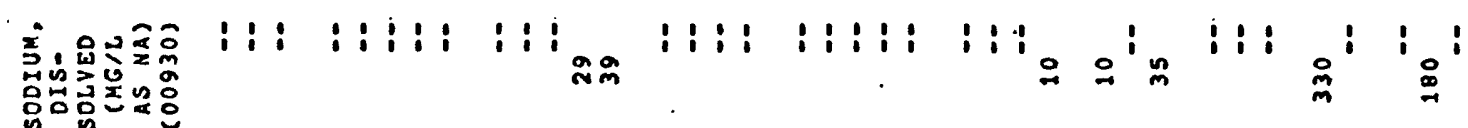

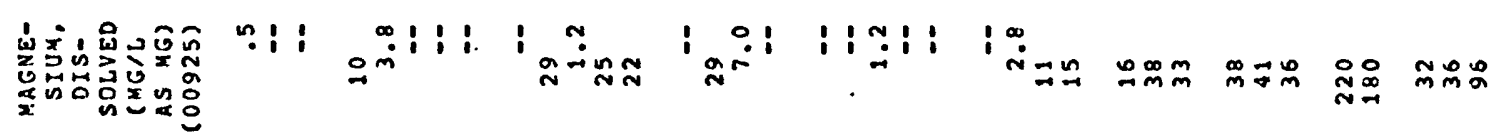

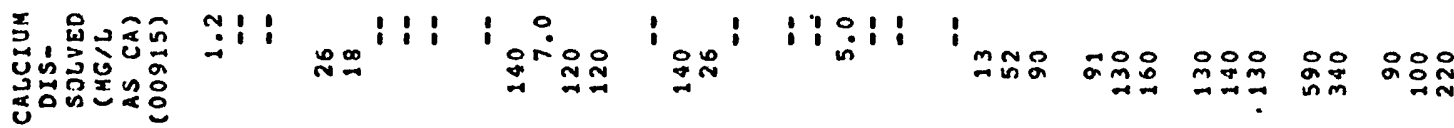

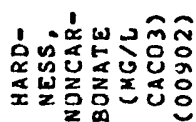

章

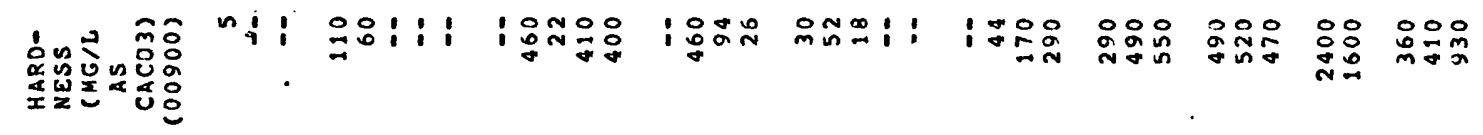

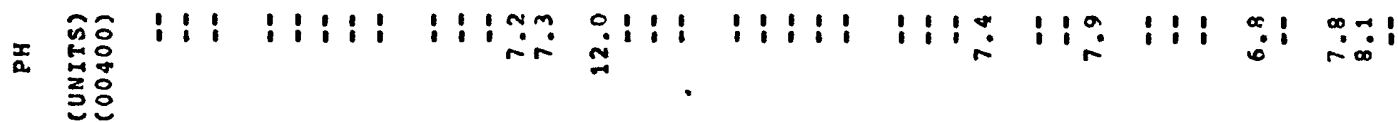

\& \&

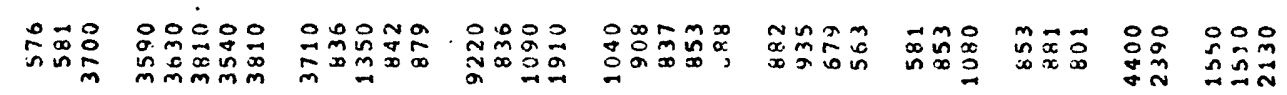
अृ

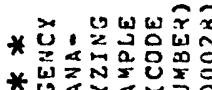

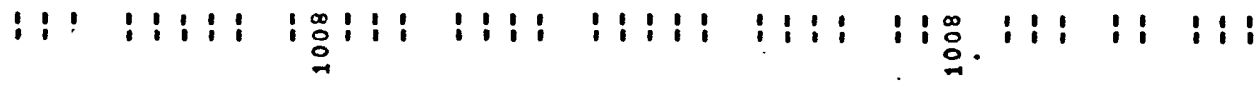

की

竞

in 0

क

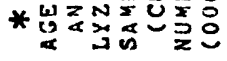

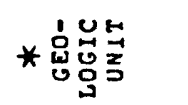

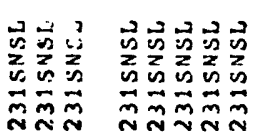

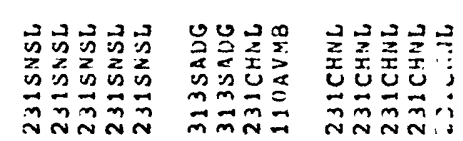

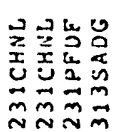

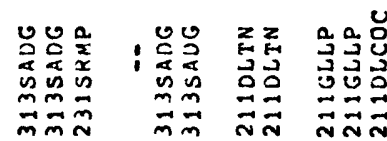

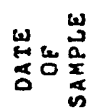

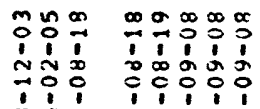

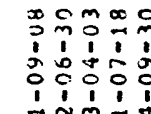

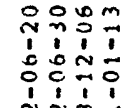

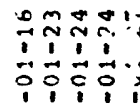

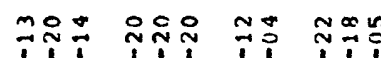

กั

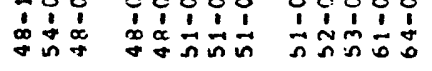

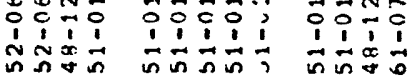

$\begin{array}{lll}0 & 0 & 0 \\ 1 & 1 & 1 \\ \text { in } & 0 & 0 \\ n & 0 & 0\end{array}$

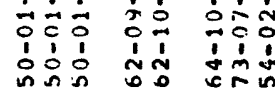

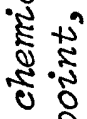

है

$\dot{0}$

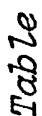
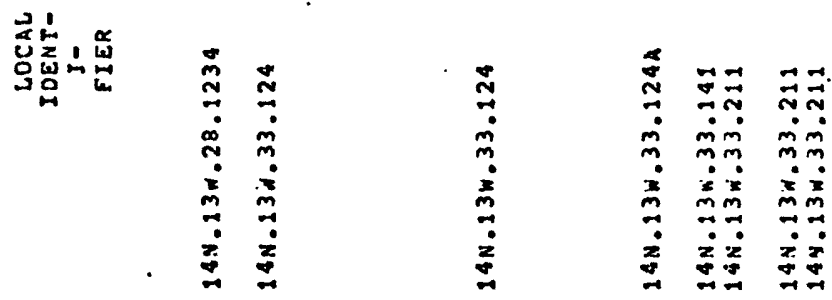

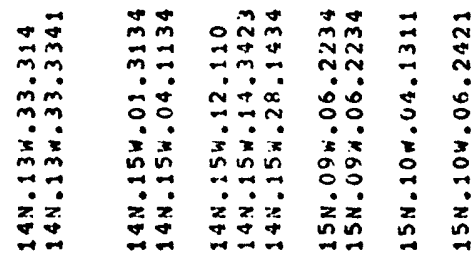




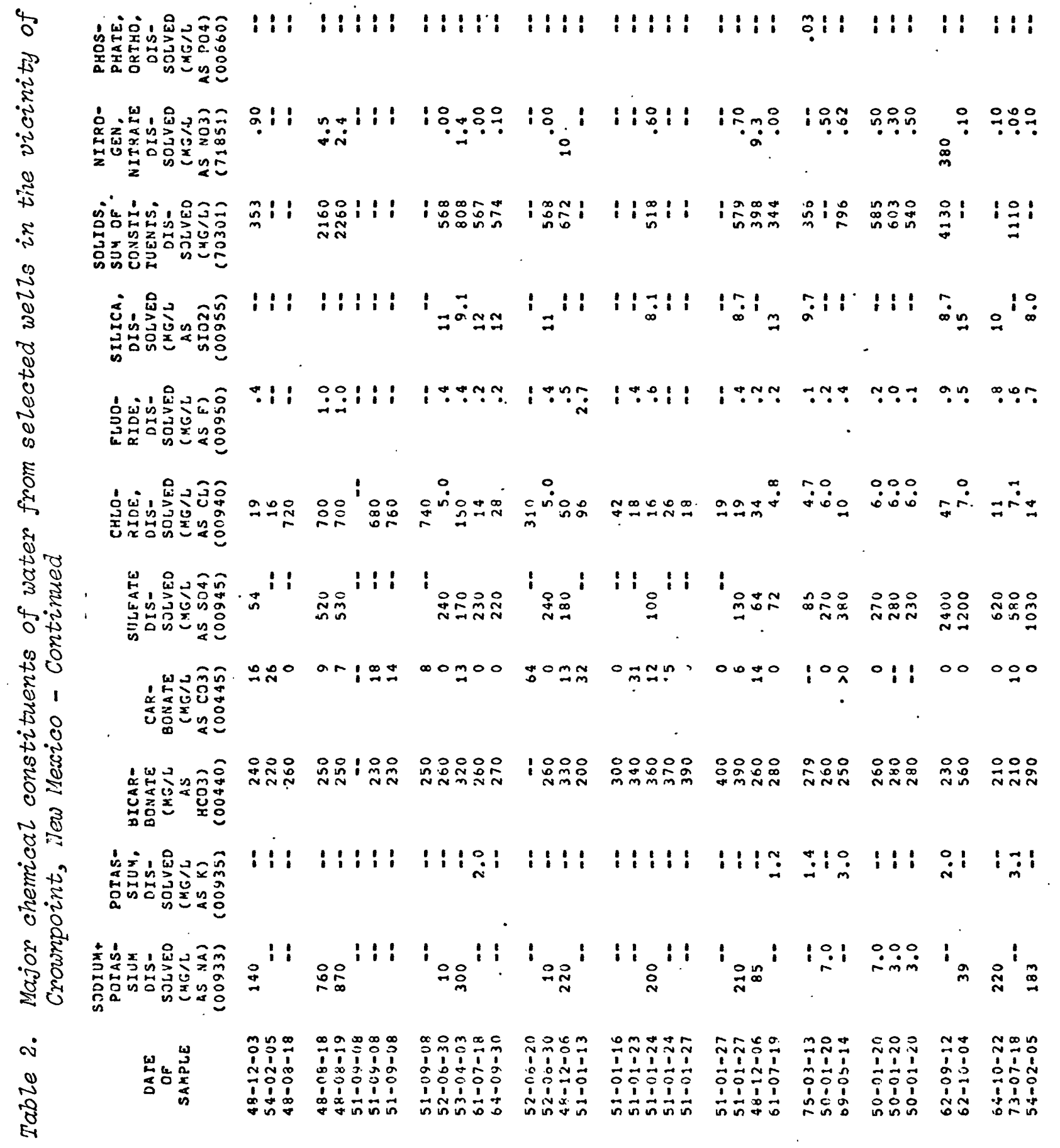




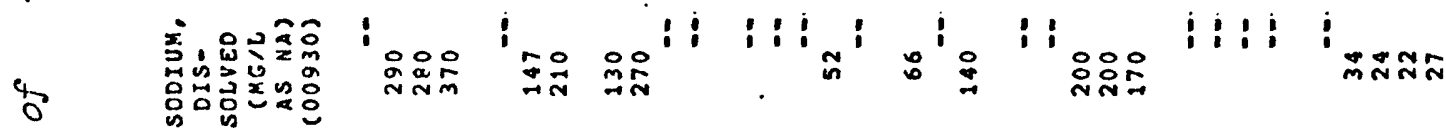

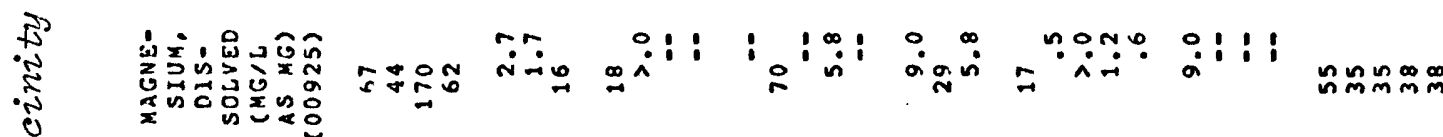
a

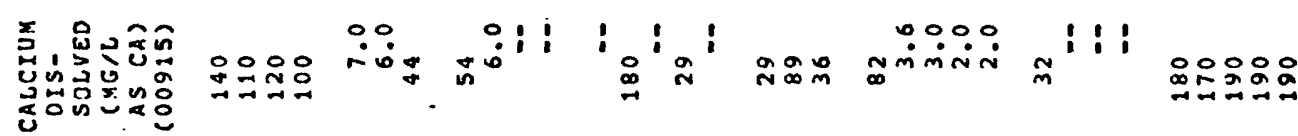
.2

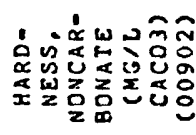

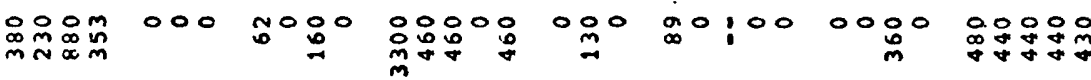

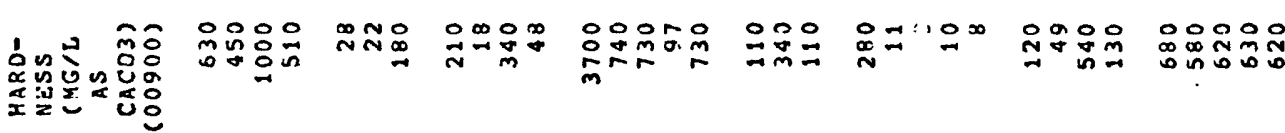

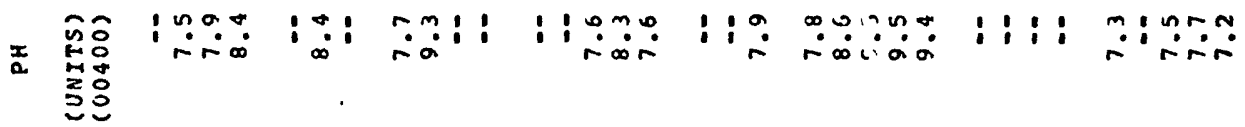

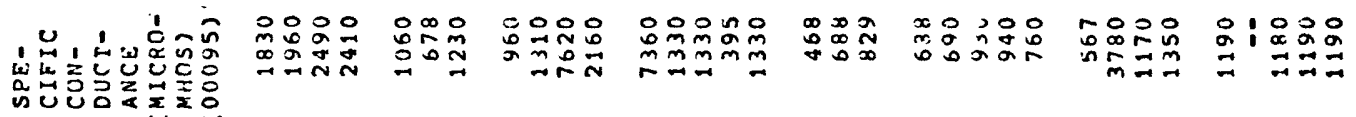

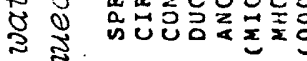

* n

है।

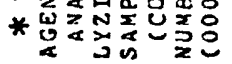

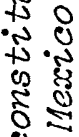

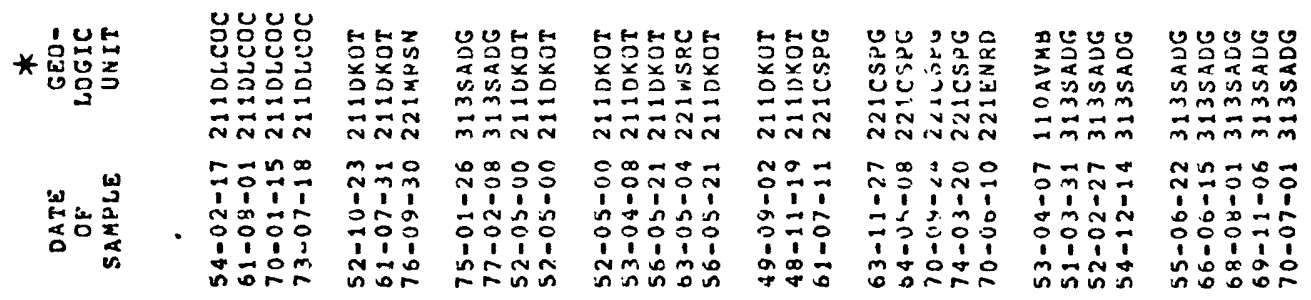
है से

N 8

है
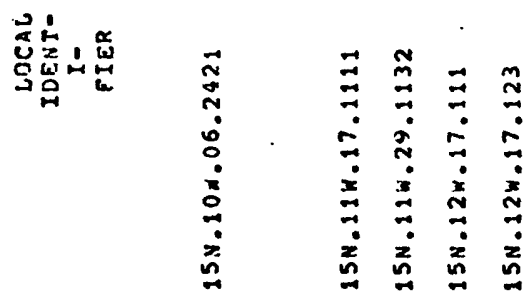
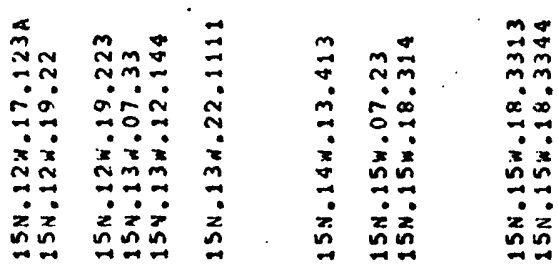


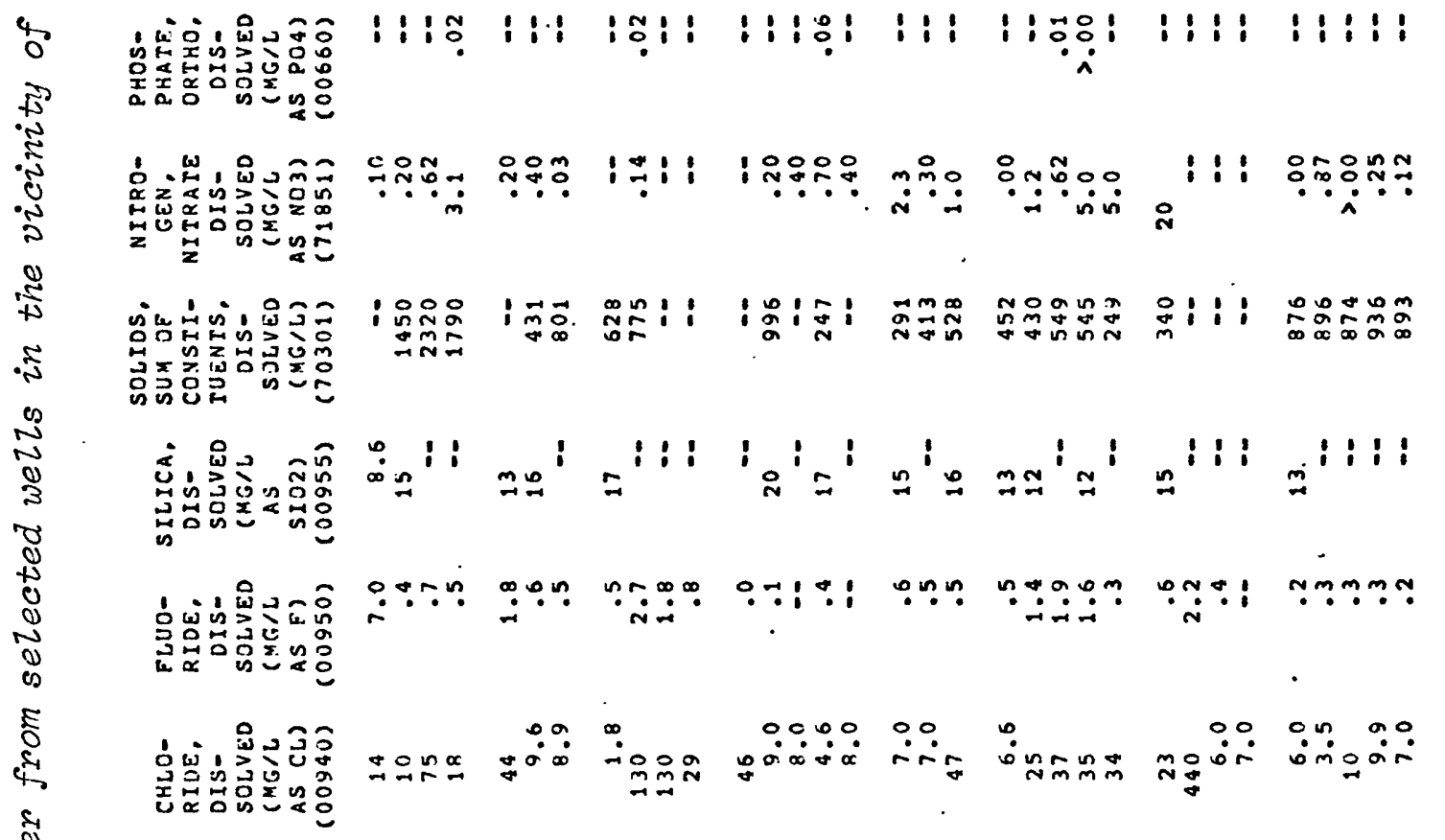

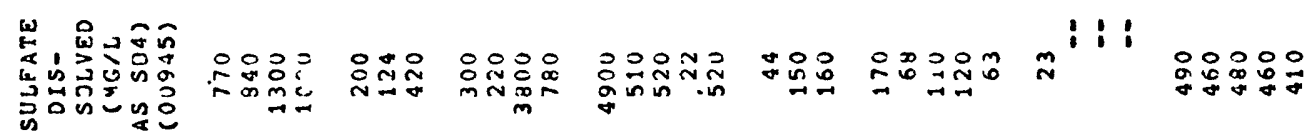

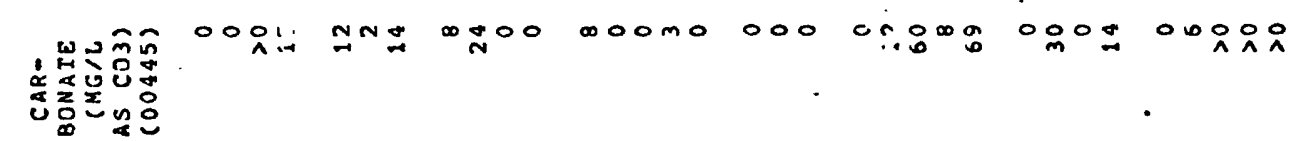

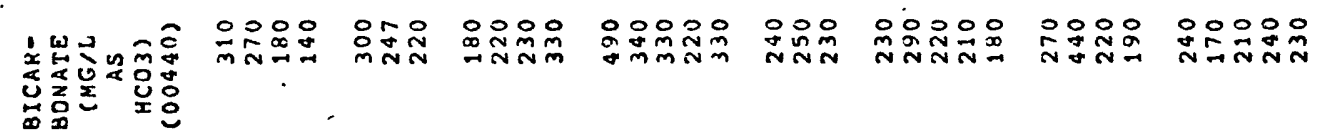

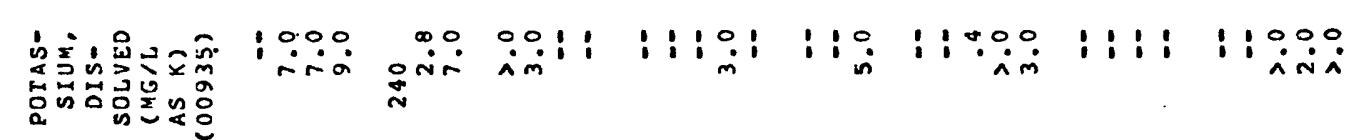

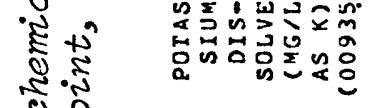

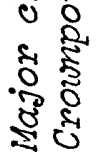

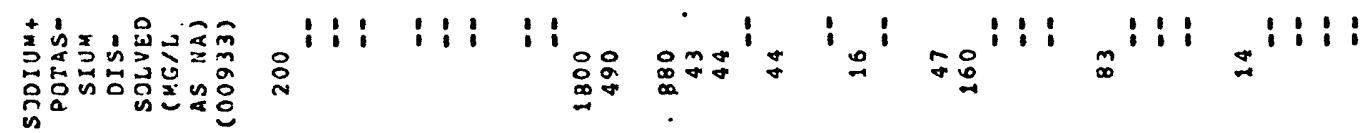
$\dot{\sim}$

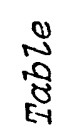

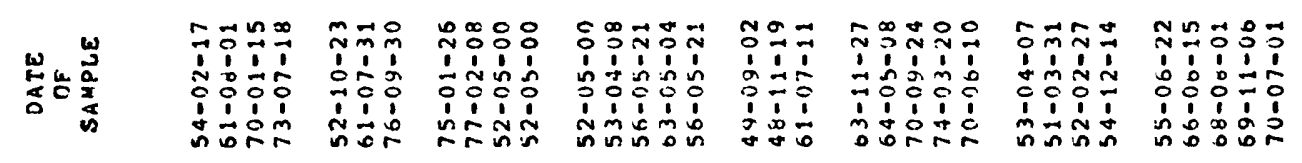




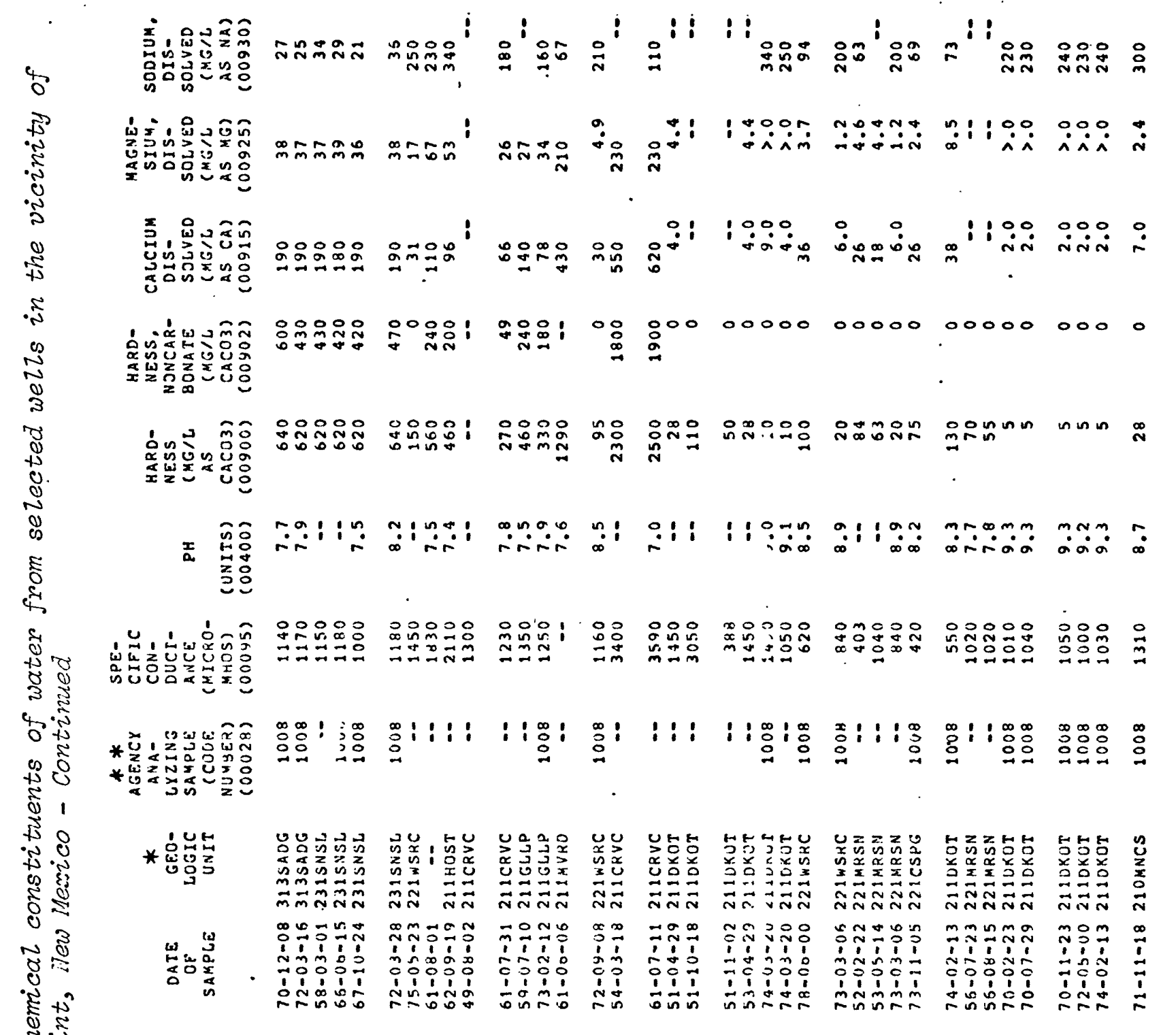

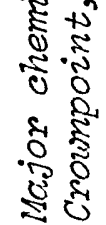

N
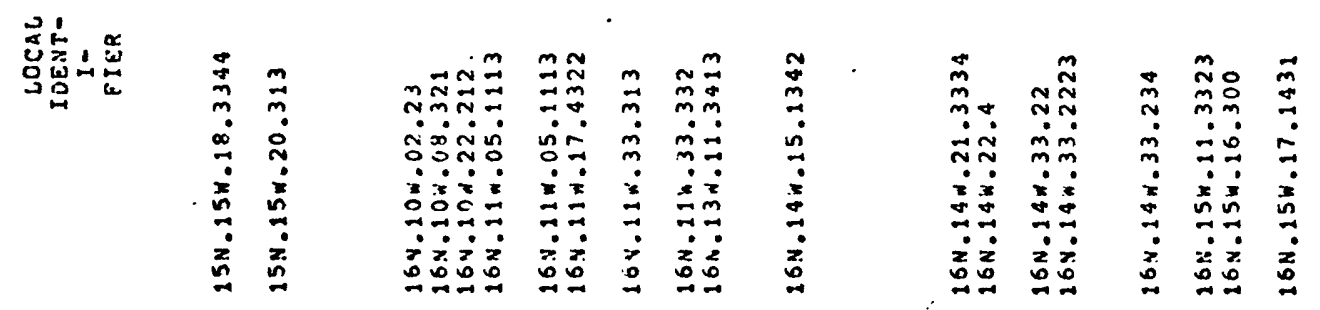

苛 


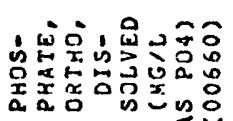
I I I I m mọn

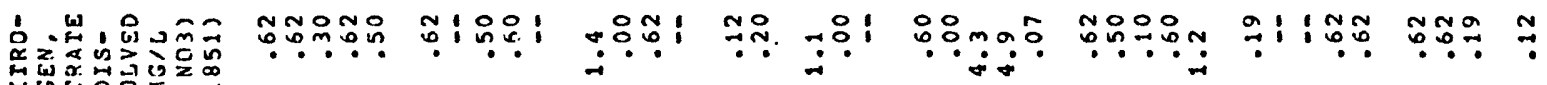

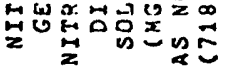

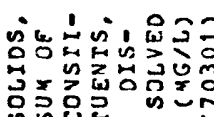

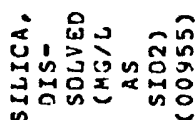

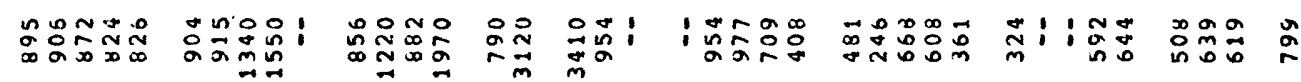

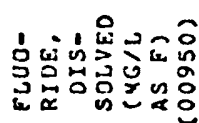

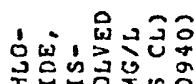

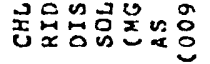

ㅁํำ

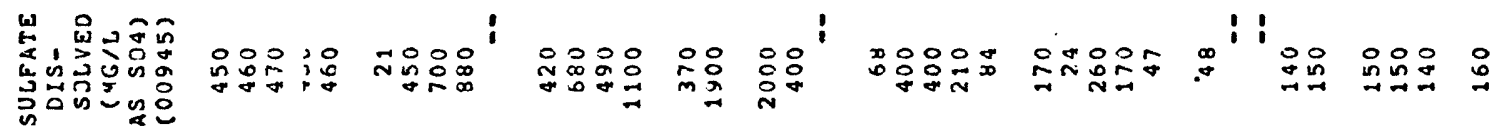

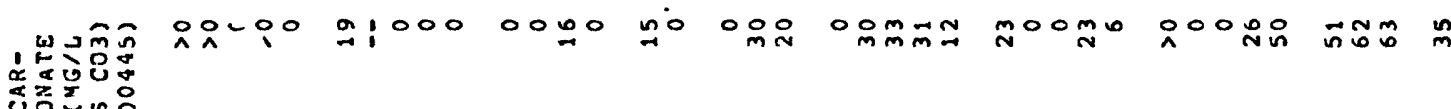

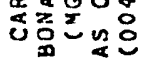

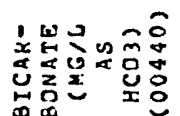

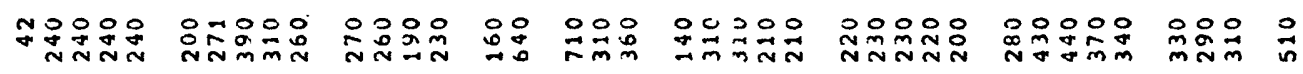

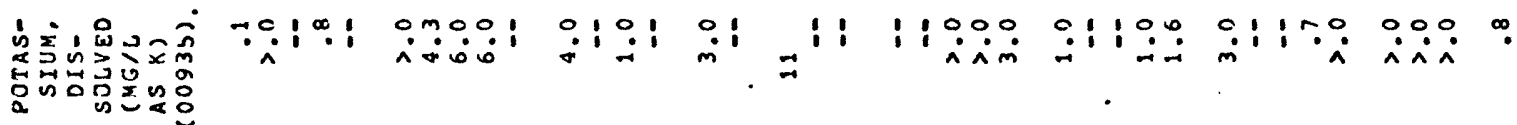

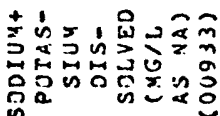

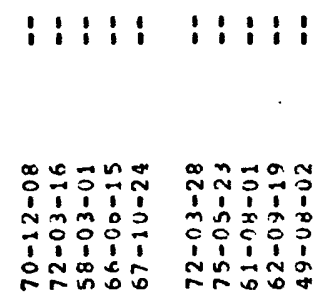

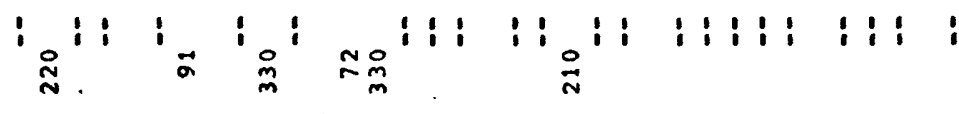
0
0
0
0
0
0

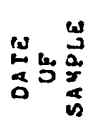

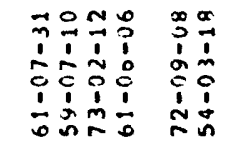

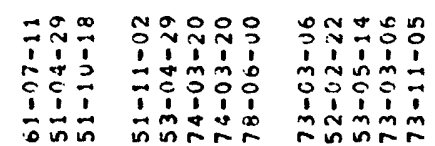

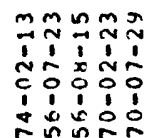

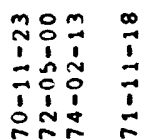




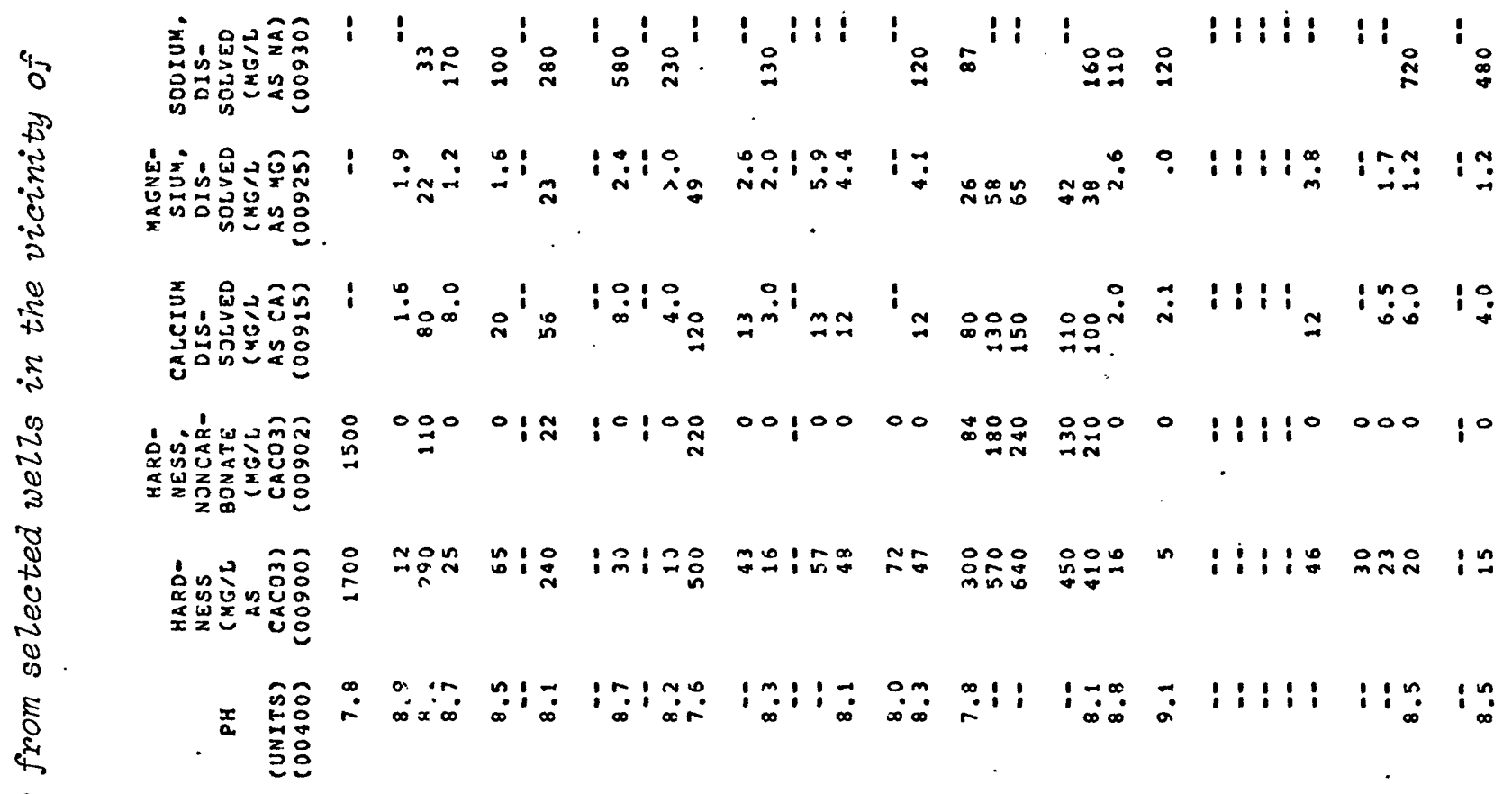

焉

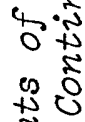

竞

in 8

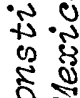

उ

종

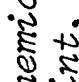

की

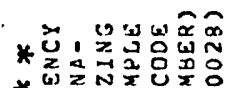

*

I

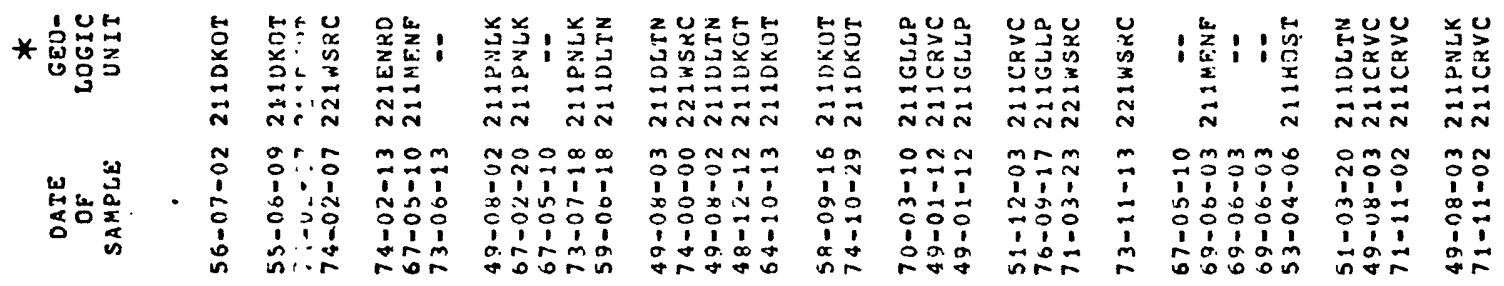

है

छ \&

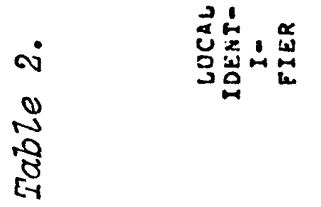

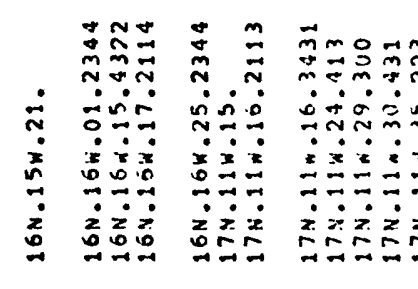

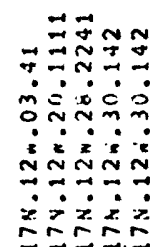

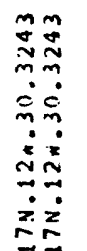

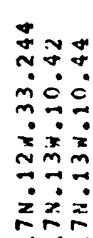

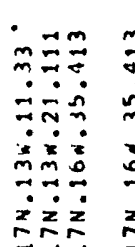

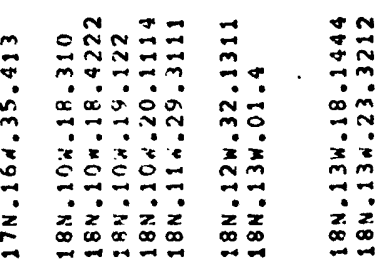




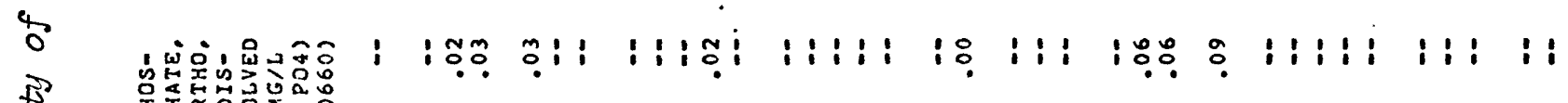
点

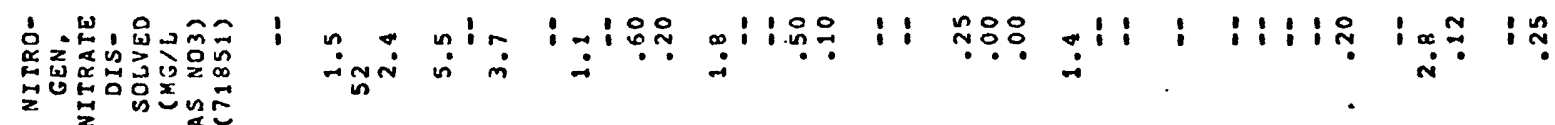

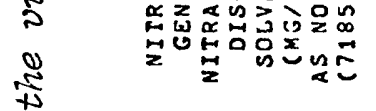

$\frac{4}{4}$

ind

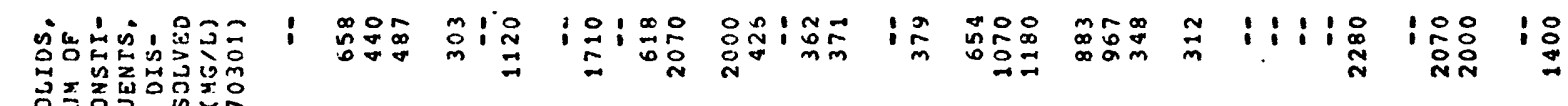

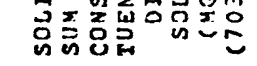

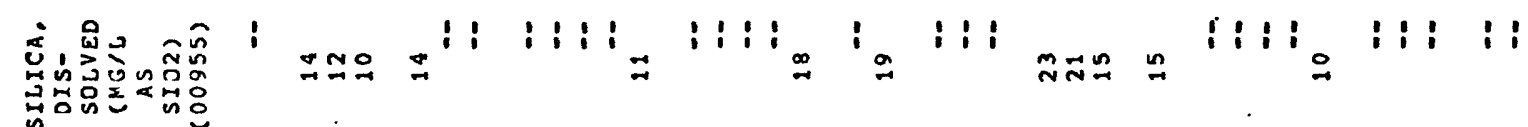

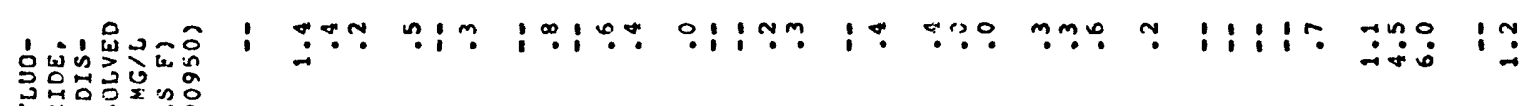

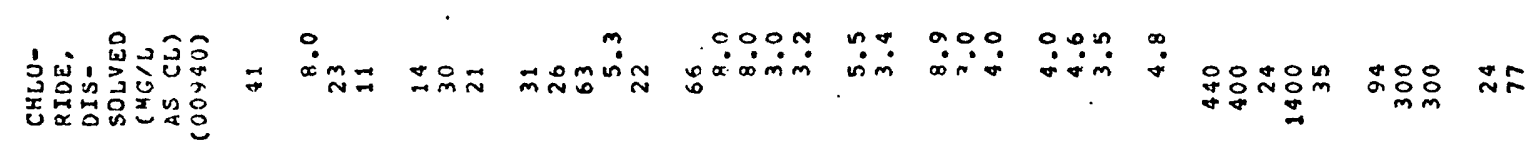

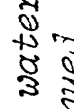

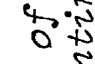
कह ठठ 8.

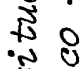

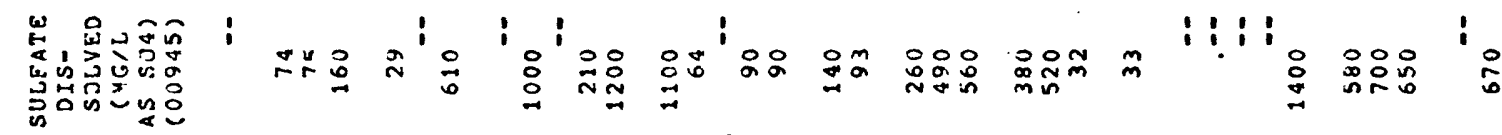

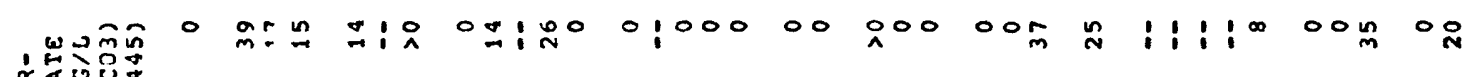

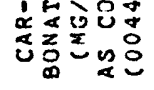

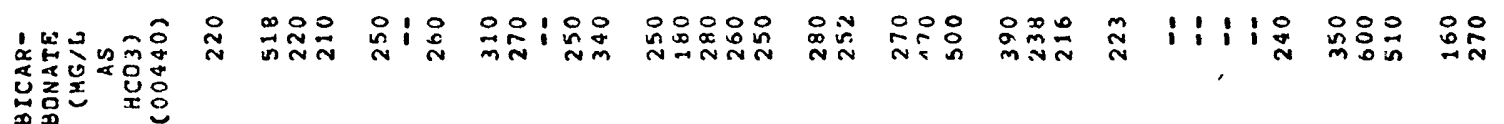
过

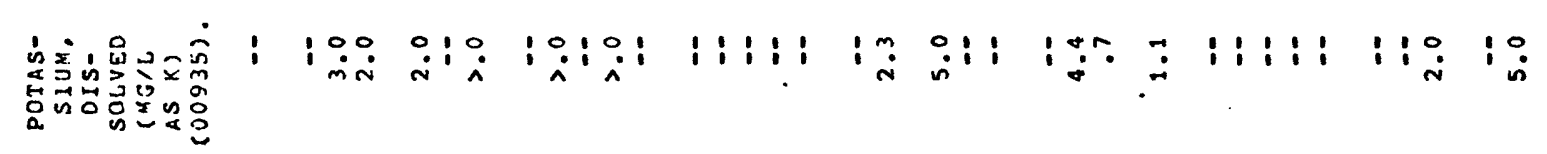

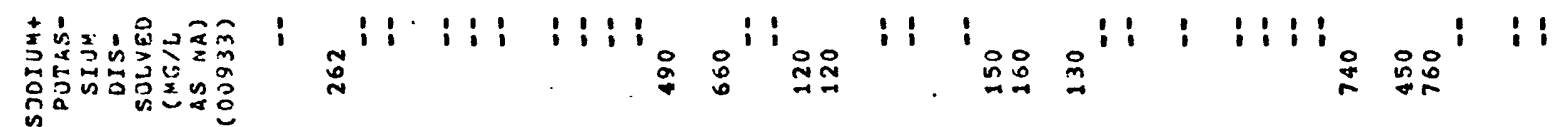
$\dot{v}$ है

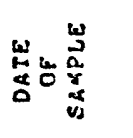

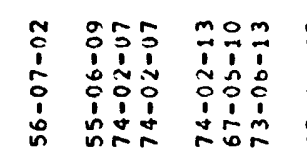

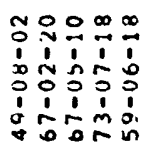

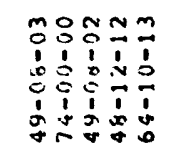

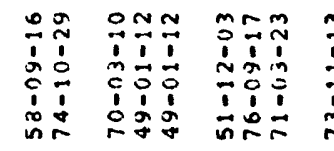

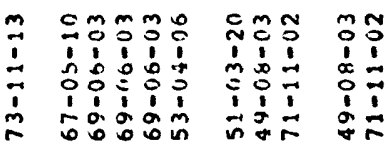




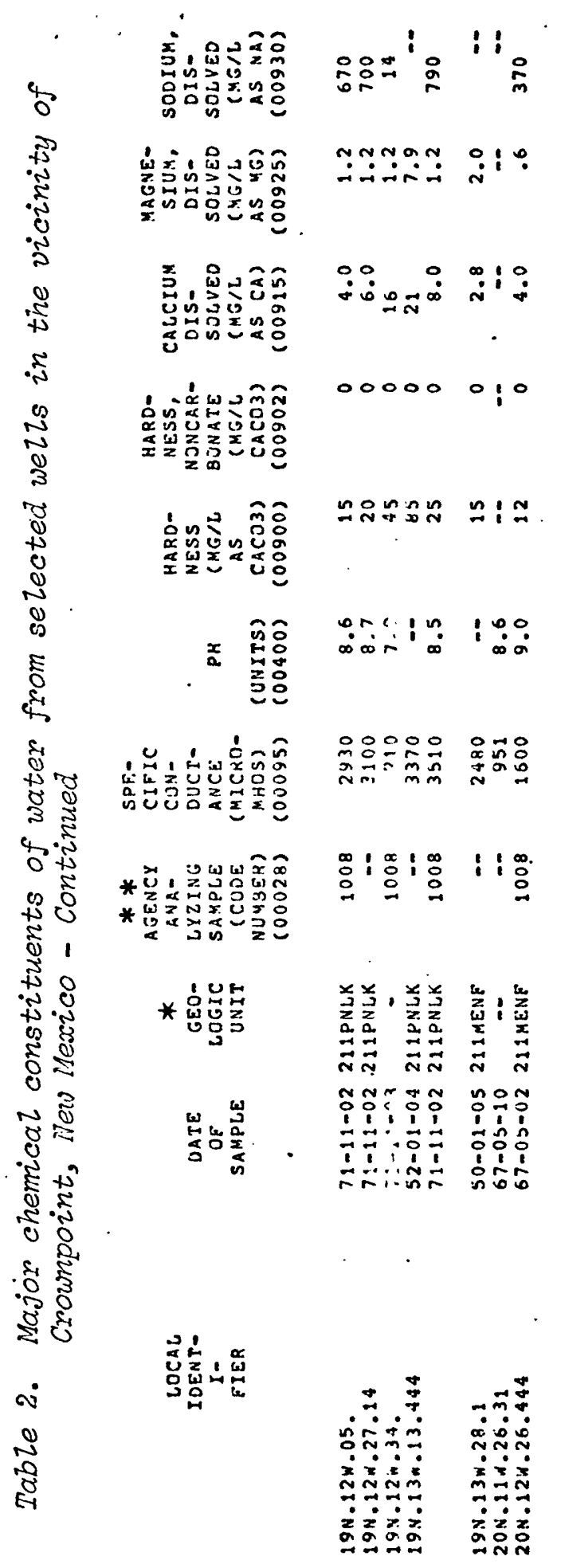




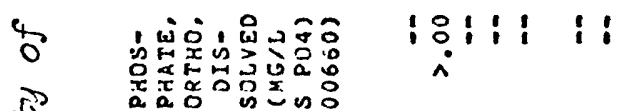

B

พ พ

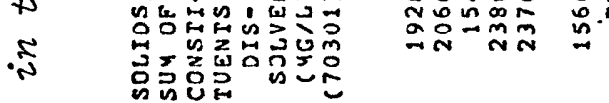

峁

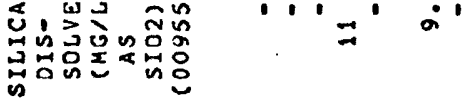

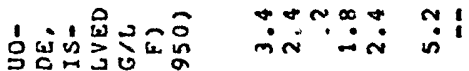

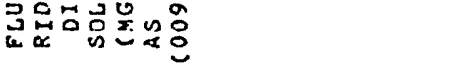

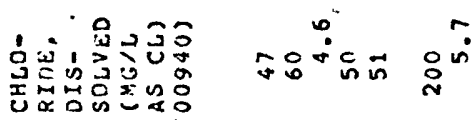

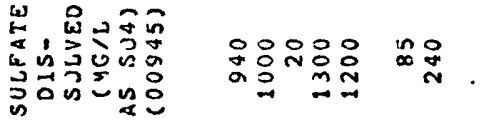

+क्ष

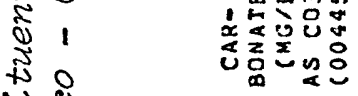

त.

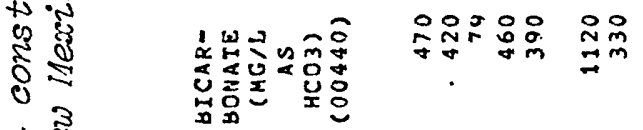

है: क्षै

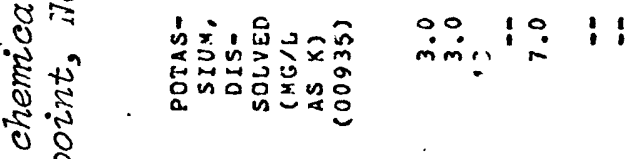

造

है

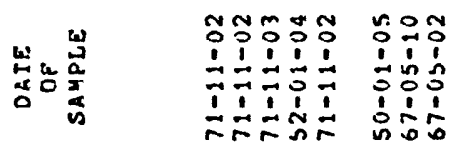

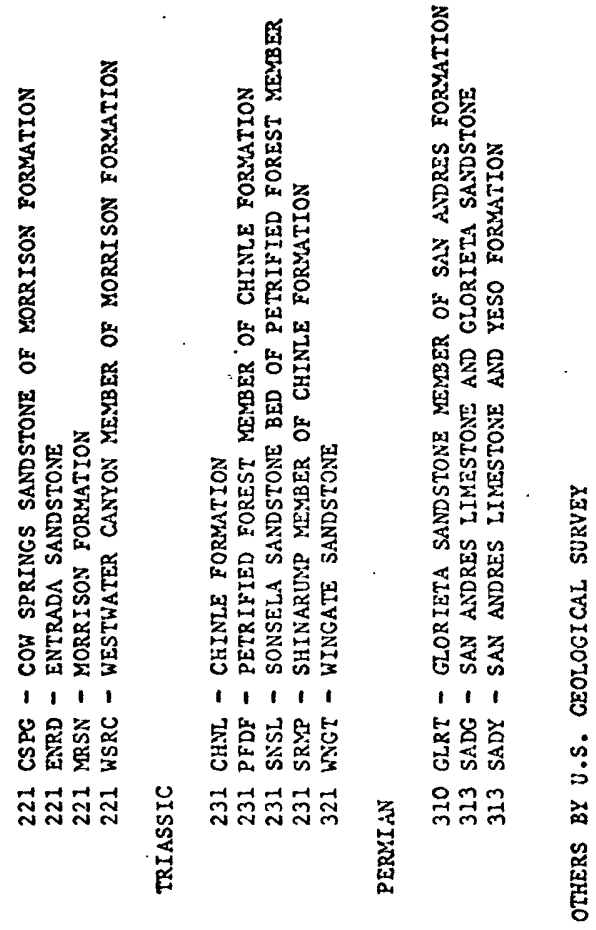

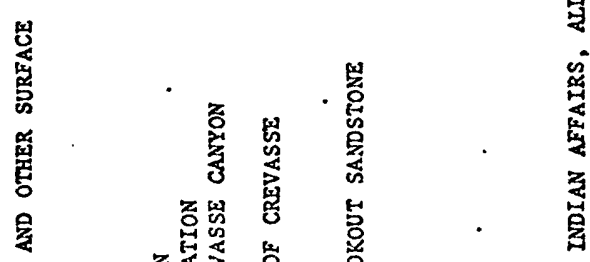

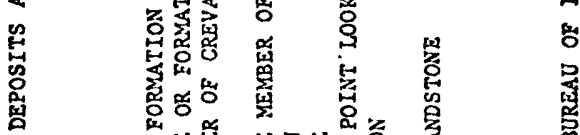

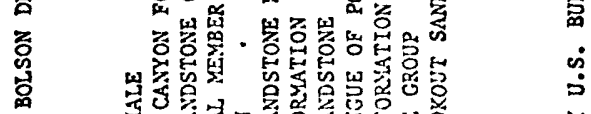

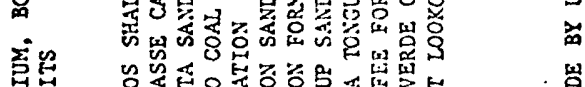

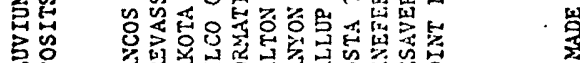

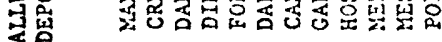

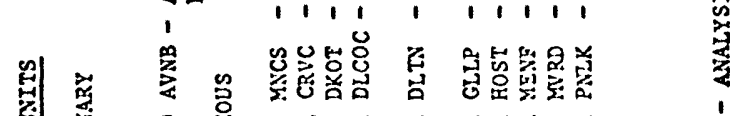

훙 


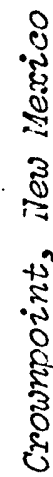

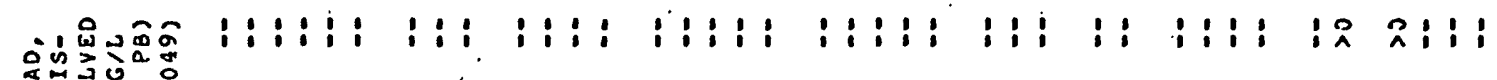

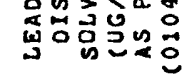

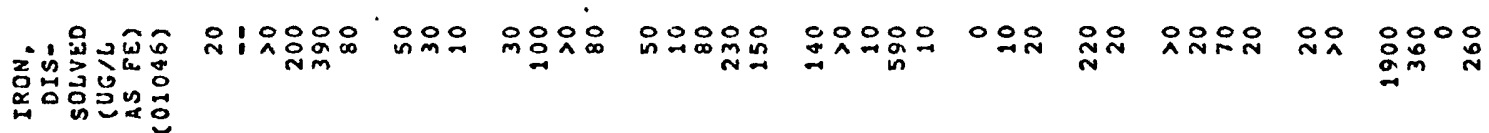

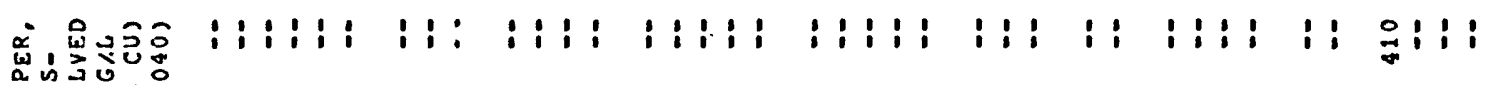

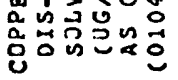

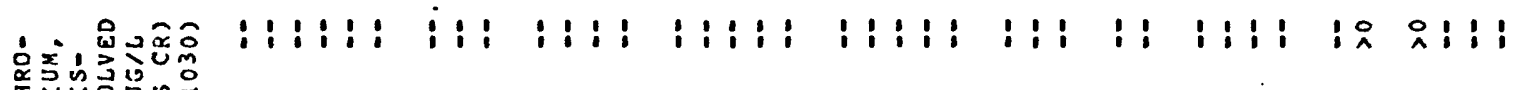

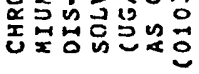

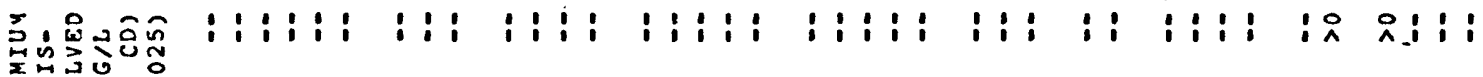

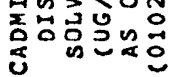

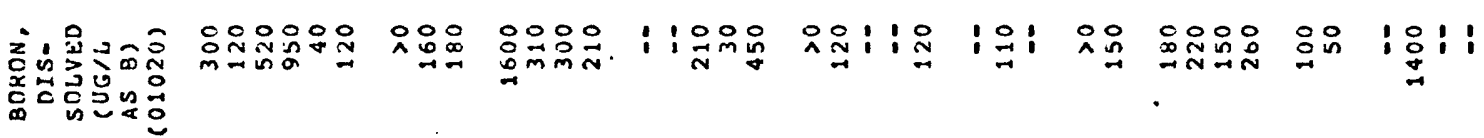

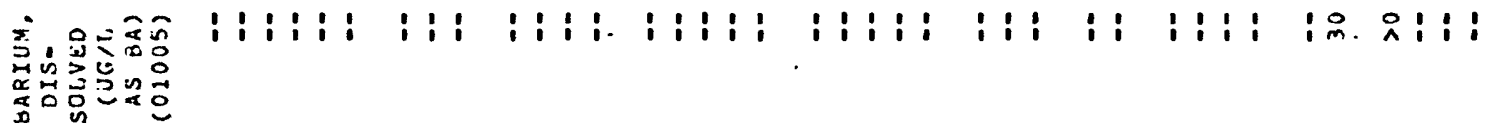

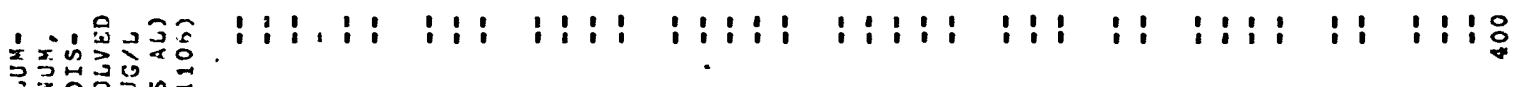

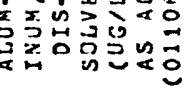

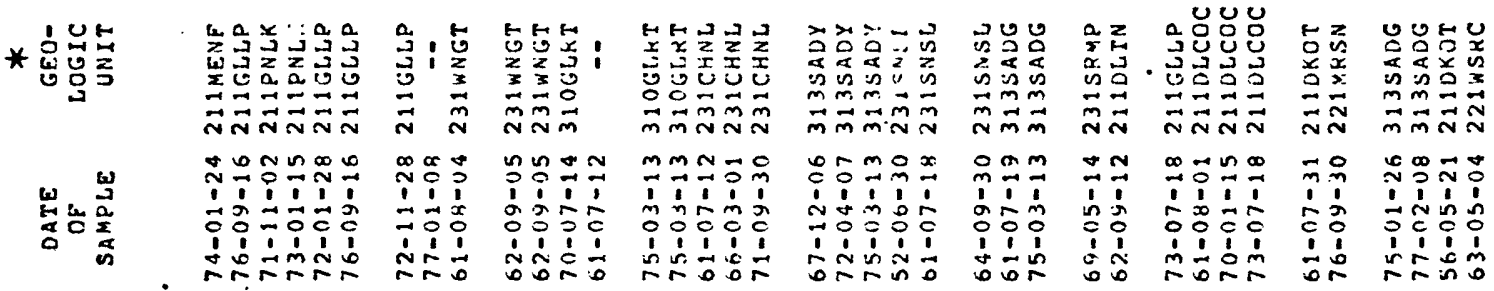

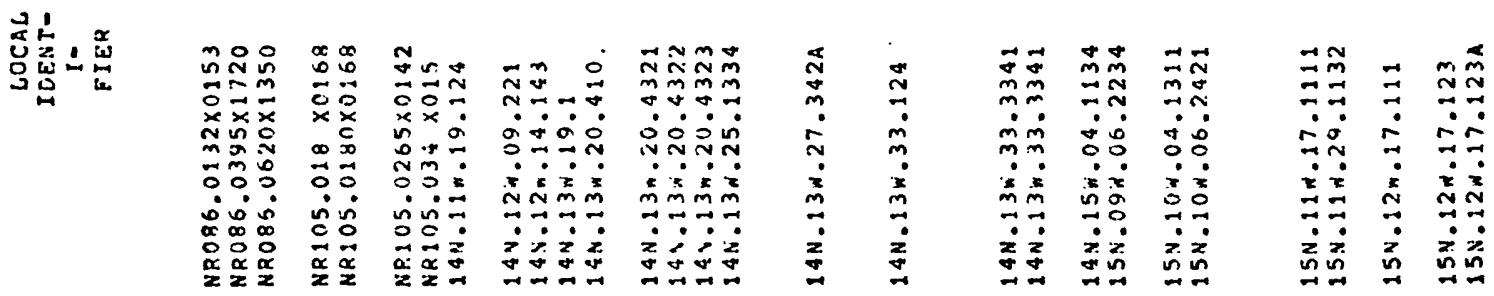
is

焉 


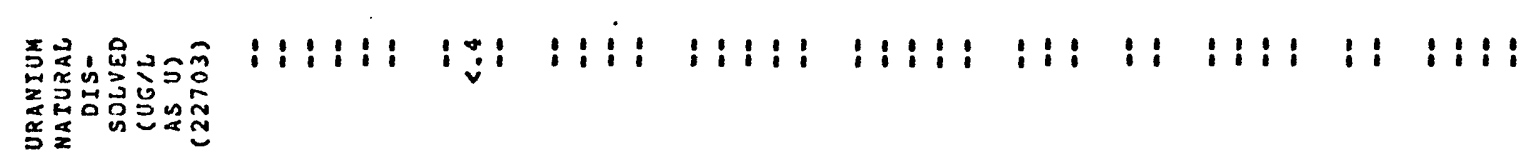

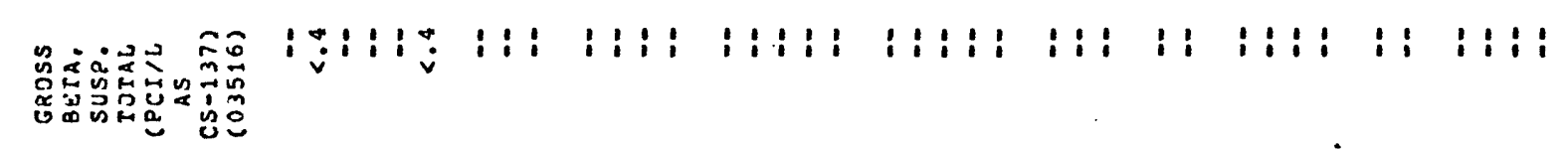

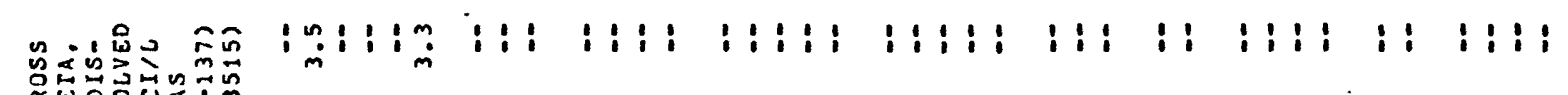

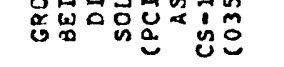

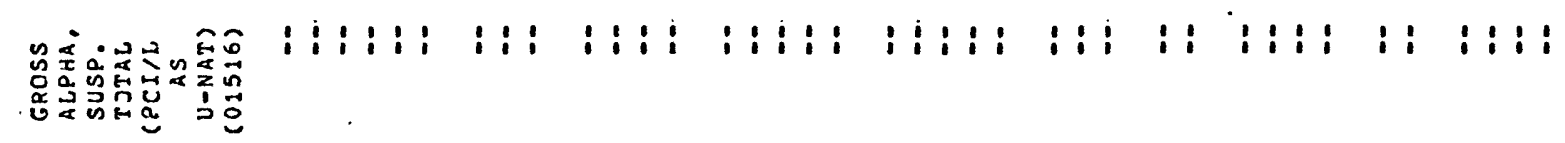

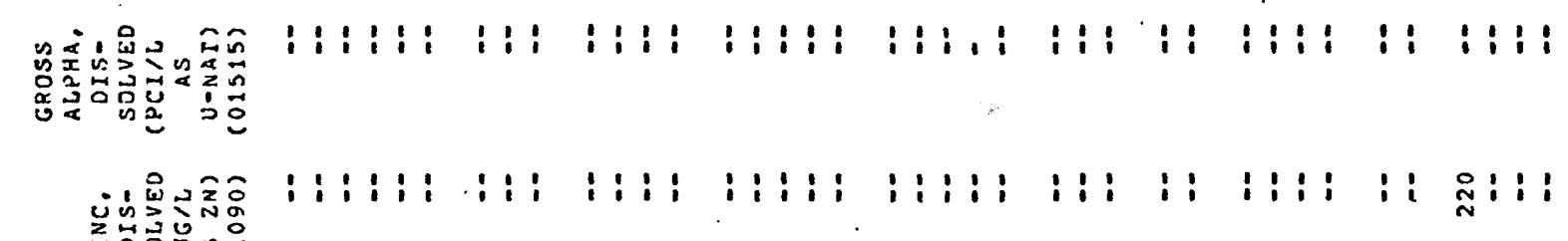
का

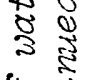

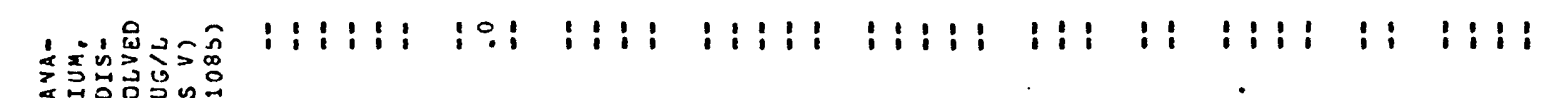
o 0 年

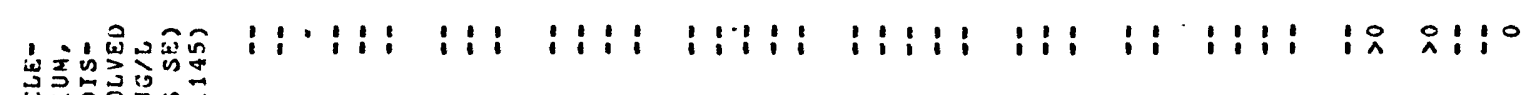
年.

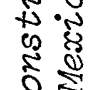

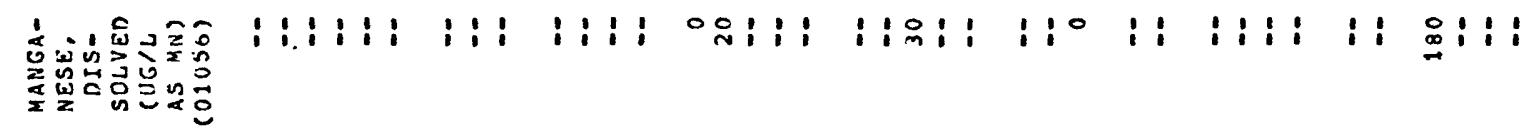

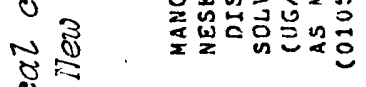

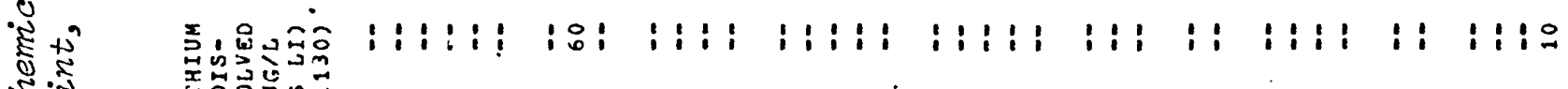

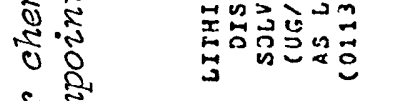

造

is

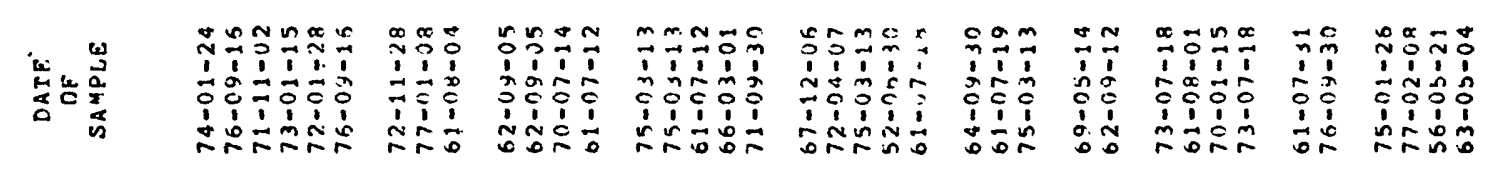
लू 


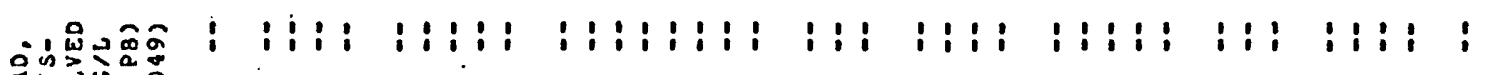

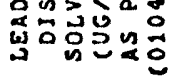

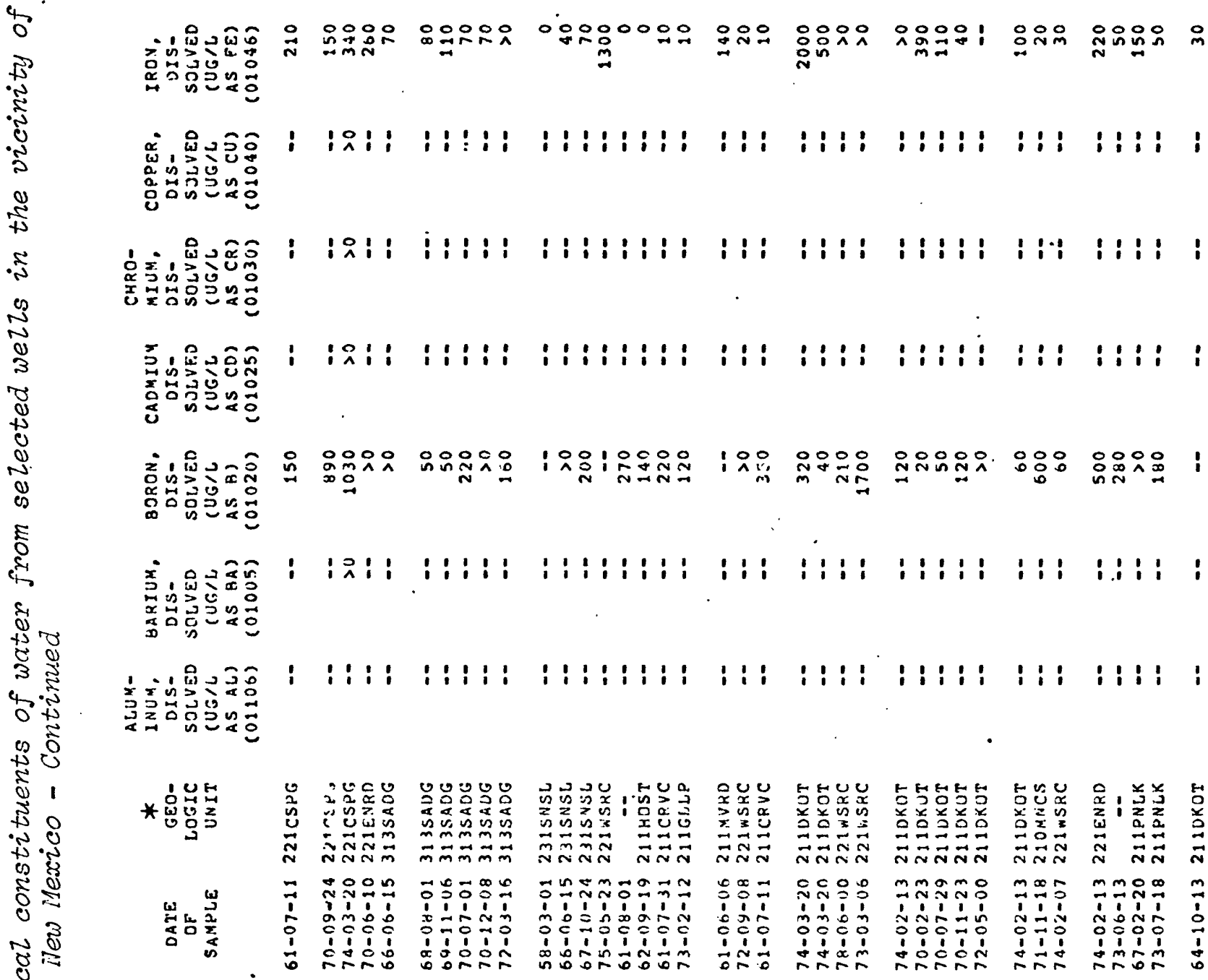

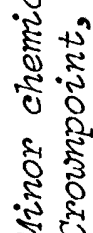

i
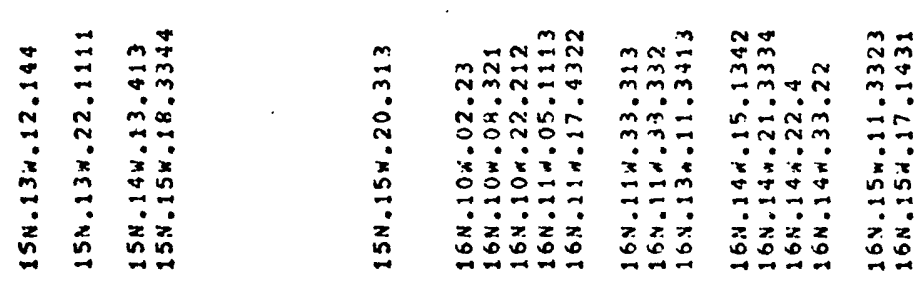

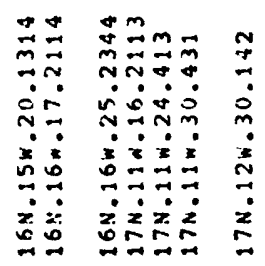




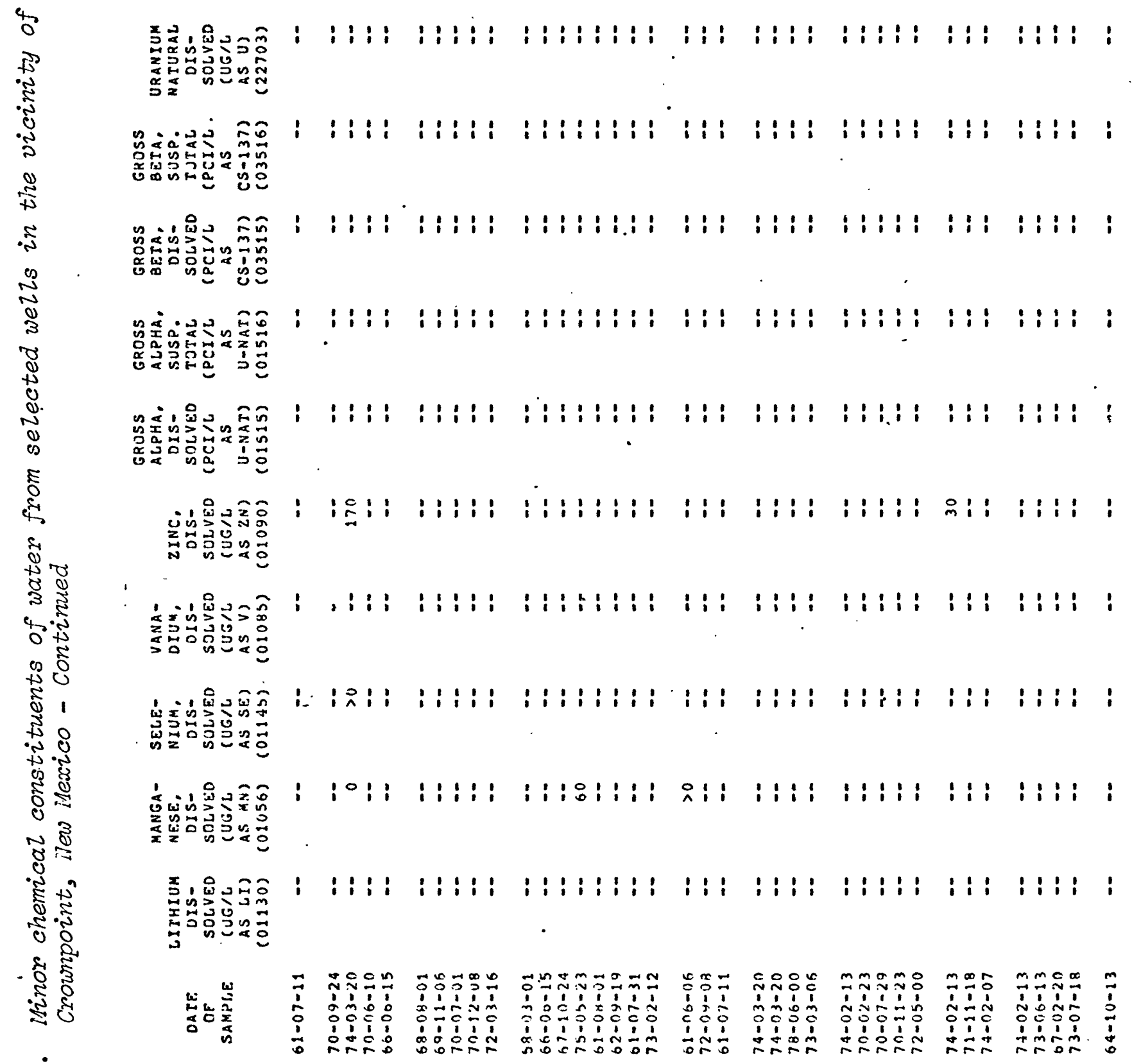

$\dot{10}$

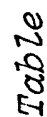




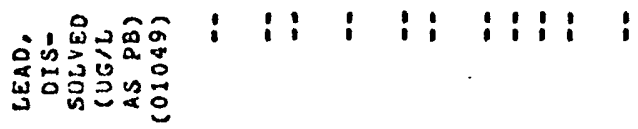

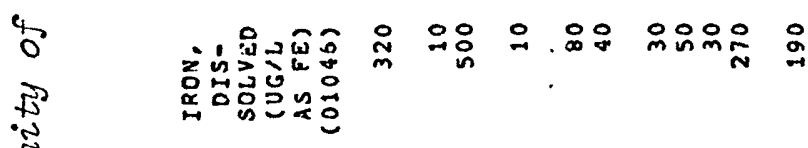

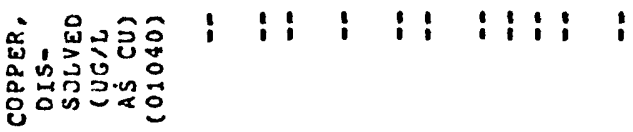

尔 1

क.

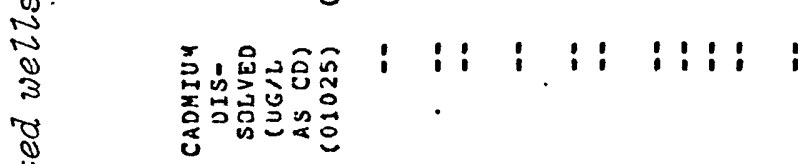

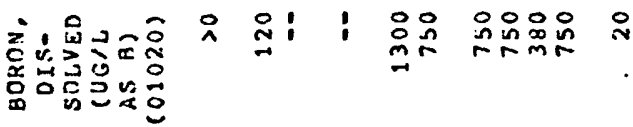

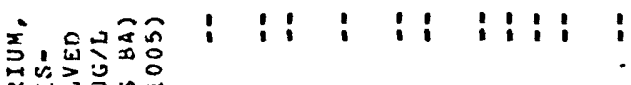

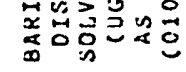

选

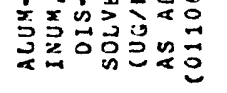

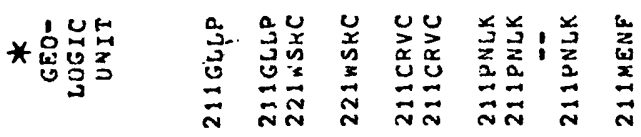

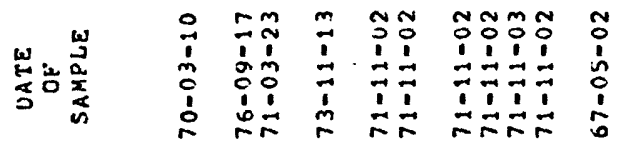

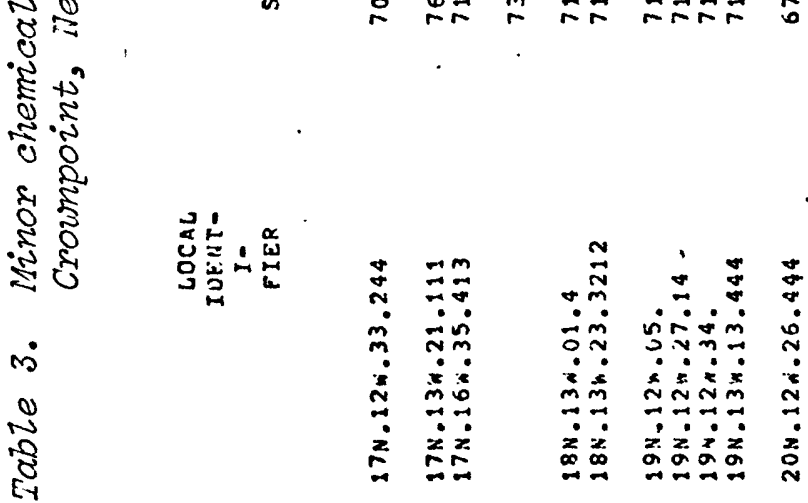




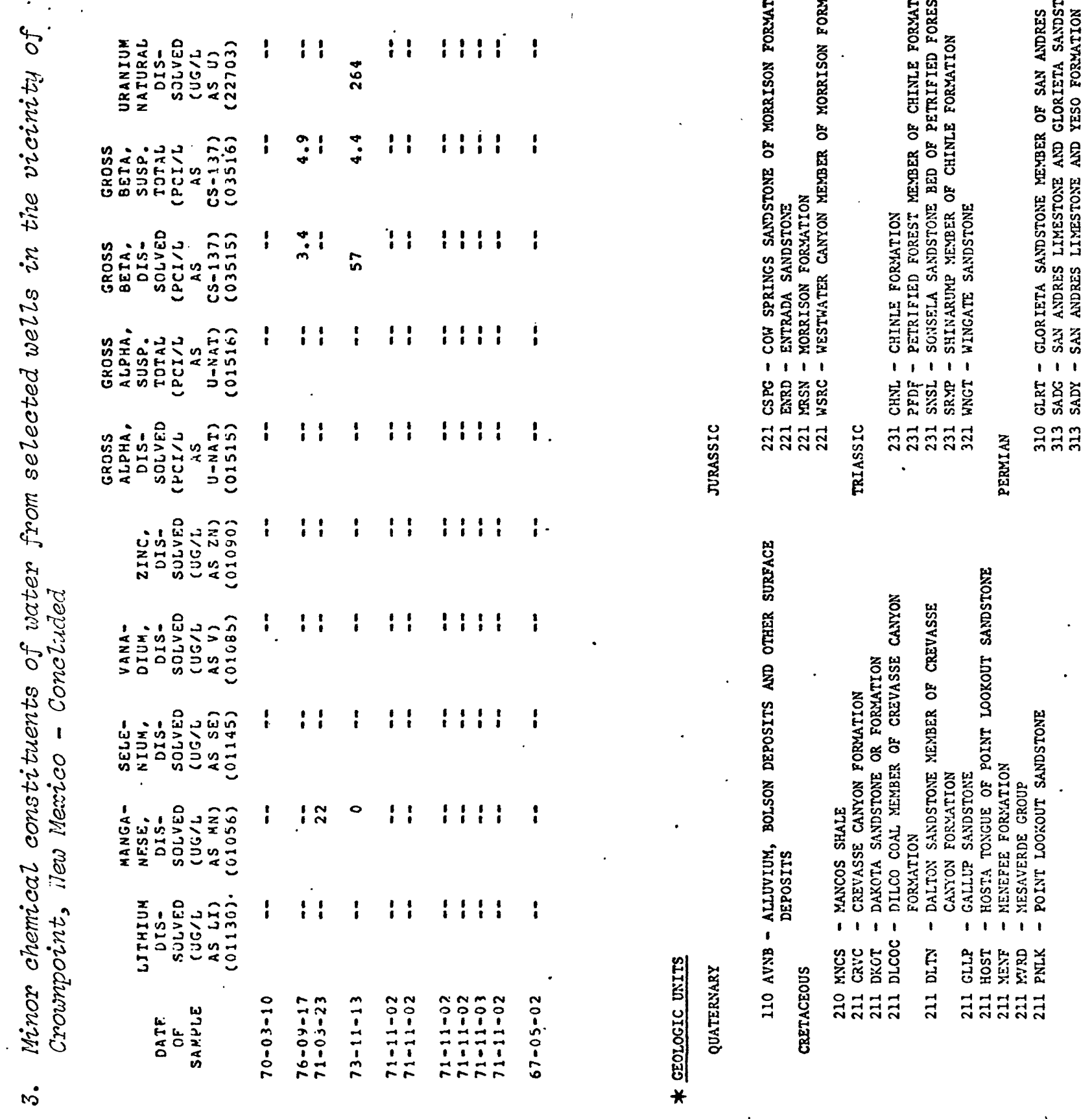

